\title{
XMM-Newton view of X-ray overdensities from nearby galaxy clusters: the environmental dependencies
}

\author{
Turgay Caglar, ${ }^{1 \star}$ Murat Hudaverdi, ${ }^{2,1}$ \\ ${ }^{1}$ Department of Physics, Yildiz Technical University, Davutpasa Campus, 34220, Istanbul, Turkey \\ ${ }^{2}$ AUM, College of Engineering and Technology, Department of Science, Dasman 15453, Kuwait
}

Accepted 2017 July 17. Received 2017 June 19; in original form 2016 October 29

\begin{abstract}
In this work, we studied ten nearby $(\mathrm{z} \leqslant 0.038)$ galaxy clusters to understand possible interactions between hot plasma and member galaxies. A multi-band source detection was applied to detect point-like structures within the intra-cluster medium. We examined spectral properties of a total of 391 X-ray point sources within cluster's potential well. $\log N-\log S$ was studied in the energy range of $2-10 \mathrm{keV}$ to measure X-ray overdensities. Optical overdensities were also calculated to solve suppression/triggering phenomena for nearby galaxy clusters. Both X-ray to optical flux/luminosity properties, $\left(X / O, L_{X} / L_{B}, L_{X} / L_{K}\right)$, were investigated for optically identified member galaxies. X-ray luminosity values of our point sources are found to be faint $\left(40.08 \leqslant \log \left(\mathrm{L}_{X}\right) \leqslant 42.39 \mathrm{erg} \mathrm{s}^{-1}\right)$. The luminosity range of point sources reveals possible contributions to X-ray emission from LLAGNs, X-ray Binaries and star formation. We estimated $\sim 2$ times higher $\mathrm{X}$-ray overdensities from galaxies within galaxy clusters compared to fields. Our results demonstrate that optical overdensities are much higher than X-ray overdensities at the cluster's centre, whereas X-ray overdensities increase through the outskirts of clusters. We conclude that high pressure from the cluster's centre affects the balance of galaxies and they lose a significant amount of their fuels; as a result, clustering process quenches X-ray emission of the member galaxies. We also find evidence that the existence of X-ray bright sources within cluster environment can be explained by two main phenomena: contributions from off-nuclear sources and/or AGN triggering caused by galaxy interactions rather than AGN fuelling.
\end{abstract}

Key words: galaxies: active - galaxies: clusters: general - X-rays: galaxies $-\mathrm{X}$ rays: galaxies: clusters - galaxies: clusters: intracluster medium - galaxies: clusters: individual

\section{INTRODUCTION}

Clusters of galaxies are formed by gravitational infalling of smaller structures, and thus they are observed to be in high density regions of the Universe. Their deep potential well retains hot gas and individual galaxies in the vicinity. The morphology and star formation rate (SFR) of such infalling galaxies change as a result of their interaction with the intracluster medium (ICM). Possible interactions and collisions between member galaxies are very likely probable. All these complexities can be effective on the galaxy evolution within galaxy clusters. The advents of the technology in space science allow us to study the evolution of galaxies in these dense and complex regions.

\section{^ E-mail: turgay.caglar@std.yildiz.edu.tr}

Several studies at different redshifts report X-ray source overdensities from galaxy clusters (e.g., Cappi et al. 2001; D'Elia et al. 2004; Hudaverdi et al. 2006; Gilmour et al. 2009; Koulouridis \& Plionis 2010; Ehlert et al. 2013). The comparison between clustered and non-clustered fields has been very successful to explain the nature of X-ray point sources. However, it is still unclear whether cluster environments suppress or enhance X-ray active galactic nuclei (AGN) activity. Koulouridis \& Plionis (2010) attempted to answer this issue by comparing X-ray and optical overdensities from 16 rich Abell clusters and reported a strong suppression within the dense $(<1 \mathrm{Mpc})$ cluster environment. Khabiboulline et al. (2014) studied low redshift clusters $(z<$ 0.2 ) and showed that AGN activity is suppressed in the rich cluster centre. Haines et al. (2012) also confirmed a similar result for massive clusters. On the other hand, radially mov- 
ing outward to the cluster outskirts, Ruderman \& Ebeling (2005) showed an enhancement of X-ray AGN activity for 51 clusters within $3.5 \mathrm{Mpc}$. This result is also confirmed for distant clusters $(z>1)$ by further investigations (Fassbender et al. 2012; Koulouridis et al. 2014, 2016; Alberts et al. 2016). A recent study found evidence that AGN emission is found to be strongly related to the richness class of the host cluster. It is understood that rich clusters suppress Xray AGN activity (e.g., Koulouridis \& Plionis 2010; Ehlert et al. 2013; Haines et al. 2012; Koulouridis et al. 2014). On the similar topic, Bufanda (2017) did not, however, find any clear correlation between AGN fraction and cluster richness based on a study of 432 galaxy clusters' data in the redshift range $0.10<z<0.95$. Therefore, The role of environment in the frequency of AGN is still an open question. A number of studies demonstrate an increased nuclear activity of the galaxies in the rich cluster environment. Martini et al. (2006) verified the existence of large low-luminous active galactic nuclei (LLAGN) populations and reported the fraction as $\sim 5 \%$ in the nearby galaxy clusters. Furthermore, Melnyk et al. (2013) reported that $60 \%$ of X-ray selected AGNs are found to be in dense environments and thus likely to reside in clusters of galaxies. Ellison et al. (2011) reported substantial evidence of increased AGN activity due to close encounters of galaxies in the gravitational potential well of the host cluster. Haggard et al. (2010) estimated approximately equal optical AGN fraction from clusters relative to the fields. Ehlert et al. (2013, 2014) found X-ray AGN fraction of 42 massive cluster centres to be three times lower than the fields. Traditional optical studies reveal a lower optical AGN fraction from clusters; the fraction for cluster and non-cluster fields is found to be $\sim 1 \%$ and $\sim 5 \%$, respectively (e.g., Dressler et al. 1999). Recent studies also confirm that optically bright AGNs are rare in cluster environments (e.g., Kauffmann et al. 2004; Popesso \& Biviano 2006).

The main astrophysical objects responsible for X-ray emission are diffuse hot gas, X-ray Binaries (XRBs) and accreting supermassive black holes (SMBHs); therefore Xray emission mechanisms are highly related to the dynamic events occurring within the galaxy. In the case of absence of very luminous X-ray sources, galaxy X-ray emission fainter than $\mathrm{Lx}<10^{42} \mathrm{erg} \mathrm{s}^{-1}$ can be produced either from star formation activities or LLAGNs. Recent studies imply that $\mathrm{X}$-ray emission from the majority of LLAGNs can be related to off-nuclear sources or diffuse emission rather than central nuclear emission (e.g., Ho et al. 2001; Ranalli et al. 2003; Ranalli 2012). On the other hand, Gisler et al. (1978) provided a correlation between star-formation and dense environments: galaxies have low star formation rates in crowd regions. Recent studies also confirmed this relationship (e.g., Kauffmann et al. 2004; Schaefer et al. 2017). Observed low star formation rates from corresponding galaxies are highly relative to the distance from central regions of clusters and associated with environmental suppression (e.g., Oemler 1974; Balogh et al. 1997; Wetzel et al. 2014). To understand properties of the star forming galaxies, some indicators have been derived from multi-wavelength surveys (Ranalli et al. 2003; Mineo et al. 2012).

In this study, we aim to understand the contribution of environment to the galaxy evolution and interaction between ICM and member galaxies. We also intend to measure $\mathrm{X}$-ray and optical density of selected galaxy clusters. There
Table 1. XMM-Newton observation logs of our sample of clusters.

\begin{tabular}{cccc}
\hline \hline Obj. Name & Obs. ID & Obs. Date & $\begin{array}{c}\text { Exp. Time (ks) } \\
\text { M1 M2 PN }\end{array}$ \\
& & & 117117113 \\
Abell 3581 & 0504780301 & $01 / 08 / 2007$ & 333328 \\
Abell 1367 & 0061740101 & $26 / 05 / 2001$ & 181817 \\
Abell 1314 & 0149900201 & $24 / 11 / 2003$ & 393933 \\
Abell 400 & 0404010101 & $06 / 08 / 2006$ & 373735 \\
Abell 1836 & 0610980201 & $17 / 01 / 2010$ & 282824 \\
Abell 2063 & 0550360101 & $23 / 07 / 2008$ & 222220 \\
Abell 2877 & 0204540201 & $23 / 11 / 2004$ & 272732 \\
Abell S137 & 0744100101 & $16 / 05 / 2014$ & 646460 \\
Abell S758 & 0603751001 & $21 / 02 / 2010$ & 444440 \\
RXCJ2315.7-0222 & 0501110101 & $22 / 11 / 2007$ & 868686 \\
Deep 1334+37 & 0109661001 & $23 / 06 / 2001$ & 565652 \\
Groth-Westphal & 0127921001 & $21 / 07 / 2000$ & 464645 \\
Hubble Deep N & 0111550301 & $27 / 05 / 2001$ & \\
\hline
\end{tabular}

is a conflict whether galaxy clustering process suppresses or enhances galaxy X-ray activity. We attempt to solve this conflict in nearby clusters by searching for X-ray and optical overdensities relative to fields. We selected a sample of several nearby galaxy clusters $(\leqslant 171 \mathrm{Mpc})$. However, in bright galaxy clusters, faint X-ray point sources cannot be detected in very bright ICMs. In that case, X-ray source number densities can be decreased. To overcome this effect, we concentrated on faint galaxy clusters with unextended ICM emission $\left(\mathrm{r}_{c}<170 \mathrm{kpc}\right)$. Our paper is organised as follows: Section 2 reviews observational samples and the data reduction process. Section 3 describes how we performed X-ray and optical analysis. Section 4 describes our measurement method for X-ray and optical overdensities. In section 5, we discuss our results in two different topics: contribution to X-ray emission from LLAGNs and star formation. Section 6 concentrate on the nature of X-ray and optical emission from individual galaxies. Finally, in section 7 , we present our conclusions. We adopt WMAP standard cosmological parameters $\mathrm{H}_{0}=70 \mathrm{~km} \mathrm{~s}^{-1} \mathrm{Mpc}^{-1}$, $\Omega_{M}=0.27$ and $\Omega_{\Lambda}=0.73$ in a flat universe.

\section{OBSERVATION AND DATA REDUCTION}

We used archival data of the XMM-Newton in our analysis, and all observational data were gathered from XMM-Newton Science Archive (XSA). In our survey, we concentrated on selecting the $X M M$-Newton observational data that were taken in full frame mode for MOS and extended full frame mode for pn. X-ray observation data logs are listed in Table 1.

The XMM-Newton data were processed by using heasoft 6.19 and XMMSAS 15.0.0 current calibration files (ccf) and summarised observation data files (odf) were generated by using cifbuild-4.8 and odfingest-3.30 respectively. We generated event files using epchain-8.75.0 and emchain-11.19 tasks from the observation data file. Rate filter is applied to the event file to clear flaring particle background. 


\section{ANALYSIS}

\subsection{Spatial and Spectral Analysis}

We applied SAS source detection algorithms to the data. Source detection was performed by using SAS task, namely edetect_chain-3.14.1. We used five different images in the super soft band $(0.2-0.5 \mathrm{keV})$, in the soft band $(0.5-1.0 \mathrm{keV})$, in the medium band (1.0-2.0 keV), in the hard band (2.0-4.5 $\mathrm{keV})$, and in the super hard band (4.5-12.0 keV) for source detection. Source detections were accepted with likelihood values above 10 (about $4 \sigma$ ) and inside an off-axis angle of $12.5^{\prime}$. Detection routine was applied for both mos and pn cameras, and the final list was combined with sas task 'srcmatch-3.18.1'. After detecting point-like sources, spectral and background files were produced by using sas task evselect-3.62. The background spectrum was extracted from an annulus surrounding the circular source region. Area of spectral files was calculated by using backscale-1.4.2. The Redistribution Matrix Files and Ancillary Response Files were produced by using SAS tasks rmfgen-2.2.1 and arfgen1.92.0 respectively. The spectra of a majority of the point sources were modelled with a single absorbed power-law. However, the spectra of several sources contain thermal emission lines that cannot be fit well by using a single power law. In that situation, we added a thermal component (APEC) to improve fitting. The average intra-galactic abundance value was fixed at 0.3 solar value in our analysis (Getman et al. 2005).

\subsection{Sensitivity of the Survey}

The sky coverage represents the survey area of the observed source and decreases with flux due to instrumental effects. Therefore, limiting flux of our survey needs to be calculated very carefully. There are a few factors that affect limiting flux, such as point spread function, vignetting, exposure time, and detector sensitivity. We calculated the sensitivity of our cameras by using sas task esensmap-3.12.1. The energy conversion factors of our samples (ECF) were calculated from rate/flux by considering hydrogen column density, photon index, and filter type of operating camera. ECF values of our samples were calculated with XSPEC model (wabs*power) with fixed photon index of 1.7 and fixed total galactic hydrogen column density value. Resulting ECF values and limit flux of our samples are presented in Table 2. Since galaxy clusters emit centrally concentrated very diffuse X-ray emission, the detection of the faint sources buried inside the ICM is not possible. To overcome this problem, we did not take into account the central region of our sample of clusters $\left(\sim 3 \times \mathrm{r}_{c}\right)$ in our analysis. Central regions of each cluster $\left(95<\mathrm{r}_{c}<145 \mathrm{~h}_{70}^{-1} \mathrm{kpc}\right)$ were calculated from King's Profile (King 1962). Due to these selection techniques, X-ray sources fainter than $1 \times 10^{-14} \mathrm{erg} \mathrm{cm}^{-2} \mathrm{~s}^{-1}$ were not taken into consideration in our analysis, and we also didn't present their properties in the appendix.

\subsection{Optical Data}

Even though a small number of red spirals and blue ellipticals are reported in the literature (e.g., Van den Bergh 1976; Masters et al. 2010), spiral galaxies are typically found in
Table 2. Detection sensitivity survey: I) Name of the galaxy cluster II) Energy conversion factor III) A total number of detected sources IV) Final source number V) Flux limit of the corresponding cluster.

\begin{tabular}{ccccc}
\hline \hline $\begin{array}{c}\text { I } \\
\text { Cluster }\end{array}$ & $\begin{array}{c}\text { II } \\
\mathrm{ECF} \\
\text { cts cm } \mathrm{erg}^{-1}\end{array}$ & $\begin{array}{c}\text { III } \\
\mathrm{N}_{T}\end{array}$ & $\begin{array}{c}\text { IV } \\
\mathrm{N}_{F}\end{array}$ & $\begin{array}{c}\text { V } \\
\text { Limit Flux } \\
\mathrm{erg} \mathrm{cm}^{-2} \mathrm{~s}^{-1}\end{array}$ \\
\hline Abell 1367 & $4.72 \times 10^{11}$ & 71 & 33 & $6.76 \times 10^{-15}$ \\
Abell 3581 & $4.91 \times 10^{11}$ & 105 & 47 & $4.07 \times 10^{-15}$ \\
Abell 400 & $3.81 \times 10^{11}$ & 62 & 33 & $6.17 \times 10^{-15}$ \\
Abell 2877 & $4.78 \times 10^{11}$ & 69 & 35 & $5.50 \times 10^{-15}$ \\
Abell S137 & $5.07 \times 10^{11}$ & 91 & 54 & $3.80 \times 10^{-15}$ \\
Abell 1314 & $4.46 \times 10^{11}$ & 111 & 39 & $5.25 \times 10^{-15}$ \\
Abell 2063 & $5.21 \times 10^{11}$ & 34 & 25 & $7.59 \times 10^{-15}$ \\
Abell 1836 & $4.17 \times 10^{11}$ & 116 & 32 & $6.31 \times 10^{-15}$ \\
Abell S758 & $4.58 \times 10^{11}$ & 130 & 59 & $2.88 \times 10^{-15}$ \\
RXCJ2315.7-0222 & $4.25 \times 10^{11}$ & 85 & 34 & $4.17 \times 10^{-15}$ \\
\hline
\end{tabular}

blue clouds, while ellipticals are usually on the red sequence (e.g., Tully et al. 1982; Kauffmann et al. 2003; Tojeiro et al. 2013). The fraction of early-type galaxies with respect to the whole galaxy population is significantly higher in clusters than in the field (e.g., Oemler 1974; Dressler 1980; Dressler et al. 1999; Kauffmann et al. 2004), whereas the number of the blue-type galaxies increases towards the outskirts of the clusters (e.g., Butcher \& Oemler 1978; Pimbblet et al. 2002). In this section, we aim to identify the colour of galaxies (blue/red) within cluster fields.

Optical counterparts of X-ray sources are identified from SDSS archive. However, we note that not all X-ray sources have optical counterparts. Also, X-ray centroid of galaxies does not always coincide with optical centroids. It is well known that major events, such as clusters mergers or tidal interactions, cause offset between X-ray and optical centre (e.g., Peres et al. 1998). Loaring et al. (e.g., 2013) demonstrated the existence of a trend between flux and positional error for $X M M-N e w t o n$ point sources and reports maximum positional error of $X M M$-Newton for faint sources as $<10^{\prime \prime}$ within off-axis angle $9^{\prime}$; moreover, the positional error of sources becomes higher at the off-axis angle $>9^{\prime}$. Owing to these assumptions, we considered optical counterpart of X-ray sources within $<6^{\prime \prime}(<4.7 \mathrm{kpc})$ radius. Then, likelihood ratio for each candidate is computed by using cross-correlation method described by Pineau et al. (e.g. 2011, and references therein). Finally, sources falling outside the likelihood ratio $<\% 50$ are assumed as background sources. We also mention that similar methods were applied to different surveys (e.g., Brusa et al. 2010; Flesch 2010; Pineau et al. 2011; LaMassa et al. 2013). We exhibit galaxy $r$ band magnitudes as a function of g-r and b-r in Fig. 1 . Magnitude values were taken from SDSS archive for the following galaxy clusters: A400, A1314, A1367, A1836, A2063, and RXCJ2315.7-0222. However, there are no SDSS observations for the rest of the galaxy clusters. To study them, we used three different catalogues to gather $\mathrm{b}$ and $\mathrm{r}$ band magnitudes of galaxies (Flesch 2010; Zacharias et al. 2005, 2013). We also note that the $K$ and extinction correction are applied to all magnitude values unless they are noted as extinction corrected. Dashed lines represent the limit value to separate blue and red galaxies (López-Cruz et al. 2004; Laganá et al. 2009). We identified a control zone using g- 

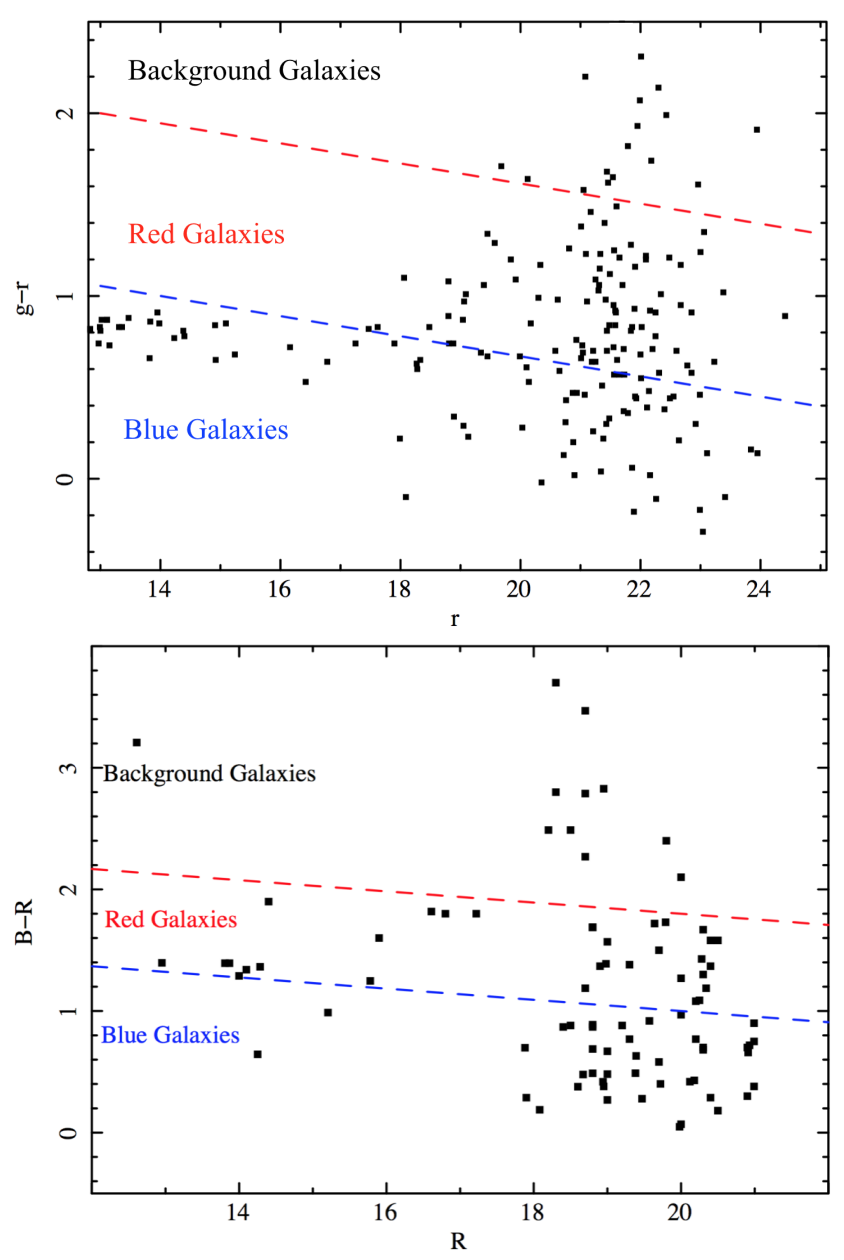

Figure 1. Colour-magnitude diagram of galaxies within our sample of clusters.

$r \pm 0.4$ limit for background galaxies. We assume that the galaxies falling outside of upper limit of the red sequence are unrelated to galaxy clusters.

\section{OVERDENSITY MEASUREMENTS}

The number of sources per unit sky area with the flux higher than $\mathrm{S}, \mathrm{N}(>S)$, is defined as ;

$N(>S)=\sum_{i=1}^{n} \frac{1}{\Omega_{i}} \operatorname{deg}^{-2}$

where $\mathrm{n}$ is number of detected sources, $\Omega_{i}$ is sky coverage for the flux of the i-th source. Fig. 2 shows $\log N-\log S$ for our samples and their comparison with Lockman hole result, which was calculated by Hasinger et al. (2001). Several studies show that cosmic variance within 2-10 keV energy range is less than $15 \%$ (e.g., Cappelluti et al. 2005; Dai et al. 2015); hence, we selected $2-10 \mathrm{keV}$ flux values for our $\log N-\log S$ measurements. In our survey, sources brighter than $\log \left(\mathrm{f}_{2-10 \mathrm{keV}}\right)=-13.5 \mathrm{erg} \mathrm{cm}^{-2} \mathrm{~s}^{-1}$ are not affected by decreasing of sky coverage, with this; we calculate X-ray overdensities at this particular flux value. At $\log \left(f_{2-10 \mathrm{keV}}\right)$ $=-13.5 \mathrm{erg} \mathrm{cm}^{-2} \mathrm{~s}^{-1}$, Hasinger et al. (2001) estimated $52 \pm 7$
Table 3. Our sample of clusters: X-ray overdensities. $\star$ : N(>S) values at $\log \left(\mathrm{f}_{2-10 \mathrm{keV}}\right)=-13.5 \mathrm{erg} \mathrm{cm} \mathrm{cm}^{-2} \mathrm{~s}^{-1}$.

\begin{tabular}{ccccc}
\hline \hline Cluster & Redshift & $\delta_{X}$ & $\begin{array}{c}\mathrm{m}_{r}{ }^{*} \\
\mathrm{mag}\end{array}$ & $\delta_{o}$ \\
\hline A400 & 0.024 & $1.67 \pm 0.75$ & 14.80 & $3.50 \pm 0.87$ \\
A1314 & 0.034 & $1.16 \pm 0.53$ & 15.24 & $5.33 \pm 1.02$ \\
A1367 & 0.022 & $1.67 \pm 0.75$ & 14.45 & $3.00 \pm 0.82$ \\
A1836 & 0.036 & $1.00 \pm 0.45$ & 15.54 & $3.10 \pm 0.64$ \\
A2063 & 0.035 & $1.00 \pm 0.45$ & 15.34 & $4.38 \pm 0.82$ \\
RXCJ2315.7-0222 & 0.027 & $0.33 \pm 0.15$ & 14.61 & $2.00 \pm 0.71$ \\
A2877 & 0.025 & $1.16 \pm 0.53$ & 14.39 & none \\
A3581 & 0.023 & $0.67 \pm 0.30$ & 14.54 & none \\
AS137 & 0.026 & $1.67 \pm 0.75$ & 14.63 & none \\
AS758 & 0.038 & $1.16 \pm 0.53$ & 15.64 & none \\
\hline
\end{tabular}

sources per degree square for the Lockman Hole Field. We calculated $53 \pm 8$ sources per degree square for Hubble Deep Field North at this flux value. X-ray source overdensities have been computed using the equation $1+\delta_{x}=N_{x} / N_{e}$ (Koulouridis \& Plionis 2010), where $N_{x}$ is the number of Xray sources brighter than $\log S\left(-13.5 \mathrm{erg} \mathrm{cm}^{-2} \mathrm{~s}^{-1}\right)$ and $N_{e}$ is expected X-ray source numbers from non-clustered fields. Optical overdensities were also calculated from the following equation $1+\delta_{o}=N_{o} / N$. In this formula, $N_{o}$ is the number of objects with the characteristic magnitude of the selected galaxy cluster within the field of view and $\mathrm{N}$ is the total number of objects from non-clustered fields with the same characteristic magnitude. Due to minimisation of projection effects, all galaxies around the cluster centre were extracted by using the method explained by Koulouridis \& Plionis (2010) (see section 3.2). Eventually, we calculated optical galaxy overdensities by using characteristic magnitude approximation with optical data. The characteristic magnitude of our clusters within range of $\mathrm{m}^{*} r \pm 2.0$ was estimated by using the following equation $\mathrm{m}^{*}=\mathrm{M}^{*}+5 \log (\mathrm{d})+\mathrm{K}(\mathrm{z})+25$ $+\mathrm{A}_{v}$, where $\mathrm{M}^{*}$ is fit parameter from Schechter Luminosity function for $\mathrm{r}$ band (Montero-Dorta \& Prada 2009), $\mathrm{A}_{v}$ is the galactic absorption, which is estimated from galactic absorption map (Schlafly \& Finkbeiner 2011), and K(z) is the K-correction factor (Poggianti 1997). We also remind that classified stars and foreground/background galaxies were not taken into consideration in our analysis. Overdensity results of our samples are presented in Table 3.

\section{DISCUSSION}

We performed data analysis for XMM-Newton observations of a sample of clusters and fields. $\log N-\log S$ was studied at limiting flux value of $1 \times 10^{-14} \mathrm{erg} \mathrm{cm}^{-2} \mathrm{~s}^{-1}$ and, we found $\sim 2$ times higher X-ray source density from our clusters compared to the values calculated in the Hubble Deep Field North and those estimated in the Lockman hole field studied by Hasinger et al. (2001) (see Fig. 2). Even in the worse case scenario according to the error limits, at least \% 35 of our point sources are cluster members. Due to minimise the influence of ICM, we did not take into account the central regions of our clusters in our analysis. Also, we studied 

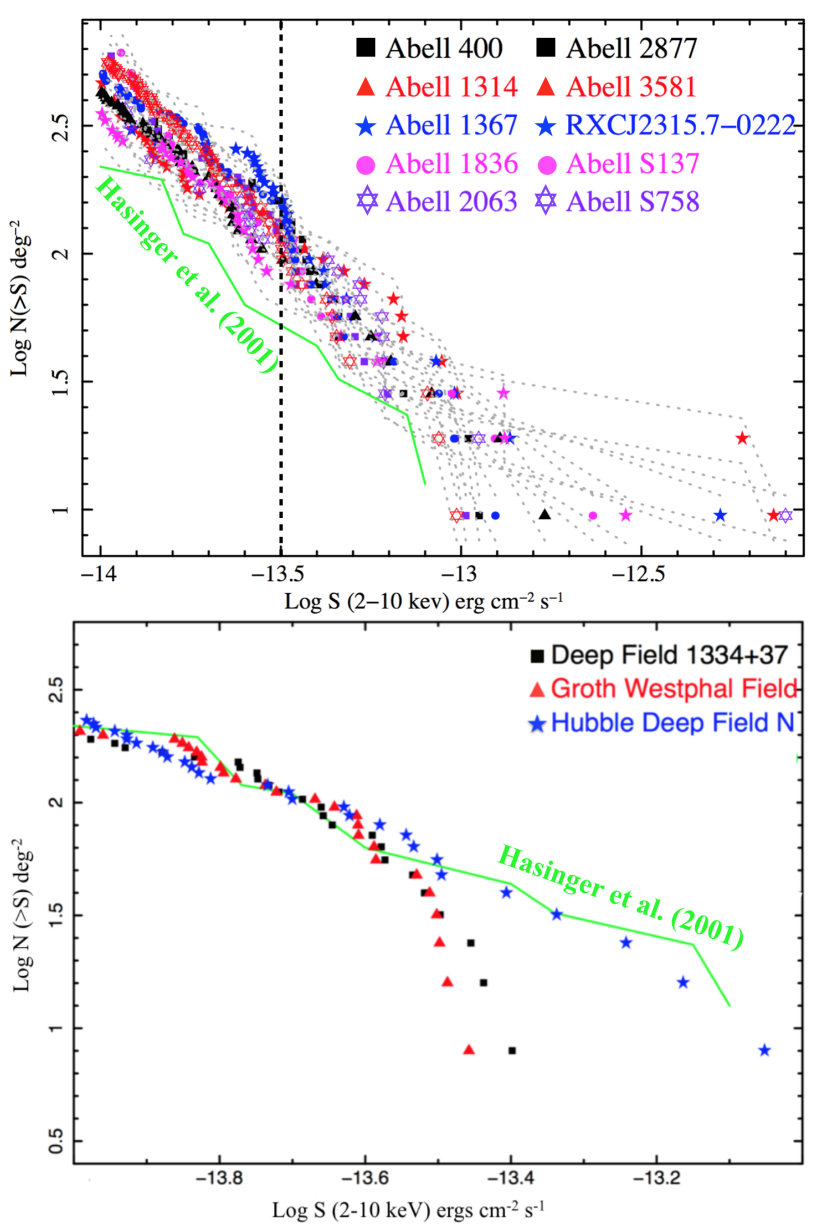

Figure 2. $\log N-\log S$ calculated in the $2-10 \mathrm{keV}$ band for our sample of clusters (Top) and our sample of fields (Bottom). The grey dotted lines represent a $1 \sigma$ statistical error. The black dashed lines demonstrate $\log (\mathrm{f})=-13.5 \mathrm{erg} \mathrm{cm}^{-2} \mathrm{~s}^{-1}$ value for visual aid, which we used in our X-ray overdensity measurements. The green lines represent Lockman Hole results, which is studied by Hasinger et al. (2001).

three different fields to enlarge our knowledge about nonclustered fields. The number counts, which were calculated in the Lockman Hole and our field samples are consistent with each other, and we confirm lower X-ray source densities in non-clustered fields than in galaxy clusters (see Fig. 2). Encouraged by this result, we calculated X-ray to optical flux ratio to understand the variety of X-ray sources detected in clusters. R-band magnitudes were compared to hard X-ray flux values and $X / O$ were calculated by using the equation $X / O=\log \left(\mathrm{f}_{X}\right)+\mathrm{C}+\mathrm{m}_{\text {opt }} \times 0.4$ (Maccacaro et al. 1998). R-band magnitudes are taken from SDSS, NOMAD and MORX catalogue, and we applied extinction correction by using extinction maps from Schlafly \& Finkbeiner (2011). Comparison between r-band magnitudes and X-ray fluxes is an advantageous method to address the condition of nuclear activity/inactivity of galaxies. Whereas AGNs tend to have $X / O>-1$ (e.g., Fiore et al. 2003), normal galaxies have $X / O$ $<-2$ (e.g., Xue et al. 2011). Besides, galaxies with $-2<X / O$ $<-1$ value can either be LLAGNs or star-forming galaxies (Park et al. 2008). However, we cannot completely explain the type of the source due to the $\mathrm{X}$-ray versus optical flux ra- tio. Therefore, we calculated X-ray to optical luminosity ratio for 40 member galaxies, and the results are given in Table A1. Matsushita (2001) studied early type galaxies and found the expected $\mathrm{L}_{X} / \mathrm{L}_{B}$ distribution of normal early type galaxies. Ranalli et al. (2005) also reported the expected $\mathrm{L}_{X} / \mathrm{L}_{B}$ distribution of late-type galaxies. We compared hard band X-ray luminosities $(2-10 \mathrm{keV})$ to blue optical luminosities to understand the behaviour of our cluster member galaxies. Absolute magnitudes were computed using the equation: $\mathrm{M}_{o p t}=\mathrm{m}_{o p t}+5-5 \log (\mathrm{d})$, where $\mathrm{d}$ is the distance in parsec unit, $\mathrm{m}_{\text {opt }}$ is apparent magnitude value. Optical luminosities in solar units were calculated by using the equation $\log \left(\mathrm{L}_{\text {opt }} / \mathrm{L}_{\odot}\right)=-0.4 \times\left(\mathrm{M}_{\text {opt }}-\mathrm{C}\right)$, where $\mathrm{C}$ is absolute magnitude of the sun in the related band. The majority of our sources has significantly higher $\mathrm{L}_{X} / \mathrm{L}_{B}$ than early type galaxies. Furthermore, \%50 of our member galaxies follow expected $\mathrm{L}_{X} / \mathrm{L}_{B}$ distribution of late type galaxies. Based on our $\mathrm{L}_{X} / \mathrm{L}_{K}$ results, we found that majority of the member galaxies is brighter in the X-rays than they are in the $\mathrm{K}$ band. The trends with $\mathrm{L}_{B}$ and $\mathrm{L}_{K}$ of $\mathrm{L}_{X}$ plots are presented in Fig. 3. Moreover, we assumed our point sources as likely cluster member and calculated luminosity values of our point sources by using cluster's redshifts. The luminosity range of our X-ray sources are found to be faint ( 40.08 $\left.\leqslant \log \left(\mathrm{L}_{X}\right) \leqslant 42.39 \mathrm{erg} \mathrm{s}^{-1}\right)$. In this luminosity range, the $\mathrm{X}$-ray emission can be produced by either LLAGNs, star formation and unresolved XRBs. We also note that the majority of X-ray sources of our survey is found to be normal or star-forming galaxies $\left(\log \left(\mathrm{L}_{X}\right)<41.00 \mathrm{erg} \mathrm{s}^{-1}\right.$ ) (see Tables $B 2, B 3, B 4, B 5, B 6$, and $B 7)$. This result implies no central nuclear activity from these sources. Early studies of the local Universe demonstrate that XRB populations dominate X-ray emission from normal galaxies (e.g., Muno et al. 2004), which can be the main X-ray emission mechanism of normal galaxies in our survey.

\subsection{The contribution from LLAGNs}

Based on our results, there is a possibility of AGN fuelling and quenching scenario in our clusters. When a galaxy falls into cluster environment under the influence of gravitational potential, the surrounding gas powers AGN (Lietzen et al. 2011), therefore, the source becomes brighter. Most of the galaxies host a black hole at the centre (Kormendy \& Richstone 1995), and possible fuelling from ICM activates inactive Black Holes (e.g., Alexander \& Hickox 2012). Besides, close encounters and collisions of galaxies are highly probable in cluster environments, where close encounters possibly cause AGN triggering (Ellison et al. 2011). Our results show the suppression of X-ray AGNs in the central regions of clusters (see Fig. 4, 5, 6). It appears that high pressurised winds from the cluster's centre affect the balance of galaxies within cluster environment and cause them to lose significant amounts of their fuel. This mechanism also explains the absence of very luminous galaxies at $\mathrm{L}_{X} \geqslant 10^{42} \mathrm{erg} \mathrm{s}^{-1}$ in nearby clusters. In this case, LLAGNs in nearby cluster environments can be related to close encounters of galaxies rather than AGN fuelling. 

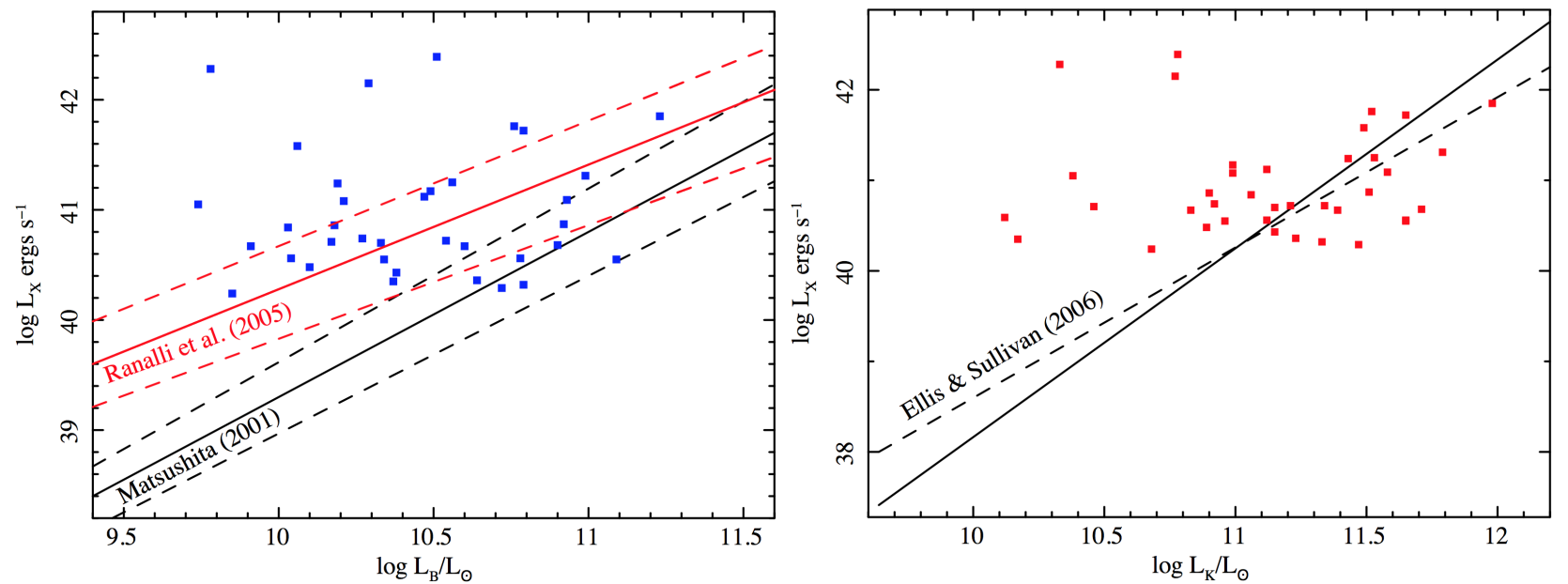

Figure 3. Top: $\mathrm{L}_{X}$ as a function of $\mathrm{L}_{B}$ for our galaxies within the cluster environment. The black solid line represents the expected distribution of early-type galaxies reported by Matsushita (2001), while the black dashed lines mark the $\pm 10 \%$ uncertainties on the relation. The red solid line represents the expected distribution of late type galaxies reported by Ranalli et al. (2005), while the red dashed lines mark the $\pm 8 \%$ uncertainties on the relation. Bottom: $\mathrm{L}_{X}$ as a function of $\mathrm{L}_{K}$ for our galaxies within the cluster environment, compared to results reported by Ellis \& O'Sullivan (2006). The black solid line shows the best-fit to their early type galaxies sample, while the black dotted line represents the expected distribution of early-type galaxies in non-clustered fields.

\subsection{Star formation}

A considerable number of recent studies reports that star formation rate increases through cluster outskirts, however star formation rates of galaxies are still lower than the field even at the viral radius of clusters of galaxies (e.g., Balogh et al. 1999; Lewis et al. 2002; Muzzin et al. 2008; Wagner et al. 2017). On the other hand, it is well known that old red galaxies dominate cluster centres (e.g., Dressler 1980); however, blue galaxies are also commonly detected in clusters. Several studies indicate that there is a significant relation between galaxy colour and star formation (e.g., Tojeiro et al. 2013), whereas blue galaxies with high SFR are bright in X-ray (e.g., Fabbiano 1982). Encouraged by this relation, we studied the colour properties of the optical counterparts of the point-like sources in our sample. In Table $B 1$, we classify our bright X-ray sources $\left(\log \mathrm{f}_{X} \geqslant-13.5 \mathrm{erg} \mathrm{cm}^{-2} \mathrm{~s}^{-1}\right.$ ) by their optical colour bi-modality by using the g-r/r or B-R/R methods (see section 3.3). We used these parameters to get indications on the nature of X-ray emission in our galaxies. We found that the number of the red and blue galaxies is approximately equal $\left(\mathrm{N}_{R} \approx \mathrm{N}_{B}\right)$ (see Fig. 1), and $\sim \% 55$ of the optical counterparts of the X-ray bright sources are identified as blue galaxies. On the other hand, a considerable number of our galaxies is found to be star-forming galaxies (see Fig. 3). Because the most massive, short-lived, newlyformed stars can become high mass X-ray binaries (HMXB) that remain bright for $\sim 10^{6-7} \mathrm{yr}$, the total X-ray emission closely tracks the star formation rate (e.g., Helfand \& Moran 2001). However, it is not possible to separate X-ray emission from HMXBs and LMXBs in distant galaxies. We note that low mass X-ray binary (LMXB) populations are quite low in late-type galaxies (e.g., Grimm et al. 2005; Fabbiano 2006). In this case, the large number of the HMXBs might cause luminous X-ray emission $\left(10^{40}<\mathrm{L}_{X}<10^{42} \mathrm{erg} \mathrm{s}^{-1}\right)$ from these sources. However, we also note that high X-ray emission from late-type galaxies $\left(\mathrm{L}_{X}>\times 10^{41} \mathrm{erg} \mathrm{s}^{-1}\right)$ can also be produced by nuclear activity and a large population of XRBs at the same time. In some cases, supernova remnants (SNRs) can make small contributions to X-ray emission at lower luminosities.

\subsection{Galaxy evolution within environment}

We studied X-ray overdensities from galaxy clusters relative to non-clustered fields. Expected X-ray source number densities were calculated in the Hubble Deep Field North, where number densities from fields are consistent with other field samples (see Fig. 2). We used SDSS archival data to obtain optical overdensities for 6 of the clusters in our sample, however, there are no SDSS observations for the remaining number of clusters. Optical galaxy overdensities were calculated in two divided areas by using characteristic magnitude method described in detail (see section 4). X-ray and optical overdensities were compared with each other to address the nature of point-like X-ray emission. As a result, X-ray overdensities are found to be significantly lower than optical overdensities in our calculations (see Table 3). However, we also point out that the X-ray overdensity of A1367 surprisingly reaches the mean optical overdensity at the outskirts of the cluster. This cluster shows an elongated shape through NW-SE direction, and two groups of star-forming galaxies are falling into the cluster's centre (Cortese et al. 2004). Recent studies imply that increased galaxy X-ray emission from cluster's field is probably caused by occurring merger events. Neal \& Frazer (2003) reported triggered AGN activity from A2255 due to a cluster-cluster merger. Also, Hwang \& Lee. (2009) studied two merging galaxy clusters and reported that cluster member galaxies show increased X-ray emission that can be related to both star formation and AGN activity. Therefore, member galaxies of A1367 are possibly triggered by ongoing merger events or in-falling of X-ray bright object that probably increased X-ray overdensity at the outskirts of A1367. In Figs 4, 5, and 6 we demonstrate 
the X-ray to optical overdensity comparisons as a function of radius. As can be seen from these figures, the optical galaxy densities decrease through outskirts of our clusters, whereas $\mathrm{X}$-ray overdensities increase through outskirts. Our results reveal that X-ray sources are suppressed within the cluster environment, and suppression of X-ray AGNs increases through cluster's centre.

\section{NOTES ON POINT-LIKE SOURCES}

In this section, we will present the results of an analysis meant to identify the main X-ray emission process (i.e., AGN or star formation) in a sub-sample of point-like sources. We present optically identified cluster members in Table $A 1$. In Table $B 1$, we present X-ray/optical properties of point sources by assuming them as likely cluster member. At low $\mathrm{X}$-ray luminosities $\left(10^{41}<\mathrm{L}_{X}<10^{42} \mathrm{erg} \mathrm{s}^{-1}\right)$, it is not clear whether X-ray emission comes from SF or LLAGN. Therefore, measurement of SFR by using different methods can be very effective to resolve the nature of X-ray emission. We calculated the SFR of point sources by using the equations defined by (Condon et al. 1992) and (Ranalli et al. 2003) respectively:

$S F R\left[M \odot y^{-1}\right]=2.5 \times 10^{-29} \times L_{1.4 G H z}\left(\operatorname{ergs}^{-1} H^{-1}\right)$

$S F R\left[M \odot y^{-1}\right]=2 \times 10^{-40} \times L_{2-10 k e V}\left(\operatorname{ergs}^{-1}\right)$

\subsection{The member galaxies}

3C 75A is identified as a pair member of NGC 1128 and the brightest galaxy of A400 (Lin \& Mohr 2004). This galaxy is elliptical and emits radio frequencies from relativistic jets (Bridle \& Perley 1984). Spectrum of this source contains absorption and thermal emission lines, and the best fit parameters are $\Gamma=1.70 \pm 0.11$, norm $_{\text {pow }}=(8.28 \pm 2.52) \times 10^{-6}$ $\mathrm{cm}^{-5}, \mathrm{nH}=0.52 \pm 0.18 \mathrm{~cm}^{-2}, \mathrm{kT}=0.8 \pm 0.05 \mathrm{keV}$, and norm $_{\text {apec }}$ $=(6.76 \pm 2.18) \times 10^{-5} \mathrm{~cm}^{-5}$. Besides, X-ray hardness ratio is measured as $-0.42 \pm 0.08$. Furthermore, X-ray to optical comparison reveals a bright X-ray emission $\left(\log \mathrm{L}_{X}\right.$ $=41.25$ ) from this source. By considering all these facts, we predict that X-ray emission mostly comes from central AGN. NGC 3860 is identified as a strong AGN (possibly triggered by super-massive black hole) by Gavazzi et al. (2011). We calculated $X / O=-1.64, \log \left(\mathrm{L}_{X} / \mathrm{L}_{B}\right)=30.68$ and $\log \left(\mathrm{L}_{X} / \mathrm{L}_{K}\right)=30.23$. Fitting the X-ray spectrum with a $(\Gamma=1.30 \pm 0.14)$, fixed column density $\left(\mathrm{nH}=1.82 \times 10^{20}\right.$ $\left.\mathrm{cm}^{-2}\right)$ and redshift $(\mathrm{z}=0.018663)$, we found a hardness ratio $(\mathrm{HR})=-0.44 \pm 0.17$ and $\log \mathrm{L}_{X}=41.17 \mathrm{erg} \mathrm{s}^{-1}$. According to these results, we claim that the X-ray emission process in NGC 3860 is due to nuclear activity, even though the source is an LLAGN. NGC $\mathbf{3 8 6 2}$ is classified as brightest cluster galaxy (BCG) (Sun 2009) and AGN (Véron-Cetty \& Véron 2010; Gavazzi et al. 2011) due to its optical properties. Our results also reveal possible low luminous AGN activity $\left(\log \mathrm{L}_{X}=41.76 \mathrm{erg} \mathrm{s}^{-1}\right.$ ) from this source (see Table A1). MCG+08-21-065 is a spiral galaxy (Sb)(Miller \& Owen 2010), and radio source (NVSS J113543+490214) is associated with this galaxy (Condon et al. 1998). Our analysis reveals very bright X-ray emission $\left(\log \mathrm{L}_{X}=42.15\right.$ $\mathrm{erg} \mathrm{s}^{-1}$ ) from this source. Therefore, MCG+08-21-065 is an AGN. Shirazi \& Brinchmann (2012) studied the optical spectrum of 2MASX J11340896+4915162 and classified this source as an AGN. On the basis of our results, we calculated $\log \mathrm{L}_{X}=42.28 \mathrm{erg} \mathrm{s}^{-1}$ and high X-ray to optical flux ratio $(X / O=-0.22)$ for this source. We confirm this source as AGN. 2MASX J15231224+0832590 is identified as a spiral galaxy (Sa) (Leaman et al. 2011). Spectral analysis of this source results in a $\Gamma=1.63 \pm 0.12$, logarithmic X-ray luminosity $\log \mathrm{L}_{X}=42.40 \mathrm{erg} \mathrm{s}^{-1}$, and hardness ratio (HR) $=0.35 \pm 0.04$. In considering these results, we identify this source as an AGN. ESO 510- G 066 is identified as a lenticular galaxy (Sa0) (Vaucouleurs et al. 1991), and show shreds of evidence of radio jets (Van Velzen et al. 2012). Spectral analysis of this source demonstrated that this source is an unabsorbed X-ray source, where $\Gamma=2.33 \pm 0.4$. The investigation reveals enhanced X-ray emission with high X-ray to optical flux/luminosity ratio $(X / O=-1.88)$. Furthermore, $\log \mathrm{L}_{X}=41.24 \mathrm{erg} \mathrm{s}^{-1}$ and hardness ratio $=-0.75 \pm 0.03$ were calculated for this source. This galaxy is located in the outskirt of the A3581, and the X-ray centroid has a positional offset $(\sim 1.5 \mathrm{kpc})$ relative to the optical centroid. We calculated the star formation rate $=34.76 \mathrm{M}_{\odot} / \mathrm{yr}$ for a given X-ray luminosity of 41.24 . This SFR measurement is in agreement with the one from the $1.4 \mathrm{GHz}$ flux (SFR $=36.98 \mathrm{M}_{\odot} / \mathrm{yr}$ ). By considering all these facts, we classify the source as a star-forming galaxy. NGC 3860B is a spiral galaxy (S) and classified as HII region-like galaxy (Gavazzi et al. 2011). As expected from H II region-like galaxies, this source appears to emit UV emission (Marcum et al. 2001). In addition, Thomas et al. (2008) studied the $\mathrm{H}_{\boldsymbol{\alpha}}$ properties of this galaxy and reported $\mathrm{SFR}=2.0 \mathrm{M}_{\odot} / \mathrm{yr}$. The SFR values we computed using the radio and X-ray luminosities are in good agreement with the UV measurements, being 3.47 $\mathrm{M}_{\odot} / \mathrm{yr}$ and $4.48 \mathrm{M}_{\odot} / \mathrm{yr}$ respectively. In conclusion, NGC 3860B appears to be X-ray normal galaxy.

\subsection{New LLAGN candidates}

In this section, we concentrate on identifying new possible low luminous AGNs from our survey. To define LLAGNs, we studied X-ray properties, $X / O$, galaxy colour and hardness ratio of point sources. Hardness ratio is defined as $(\mathrm{H}-\mathrm{S}) /(\mathrm{H}+\mathrm{S})$, where $\mathrm{H}$ is count rate in $2.0-10.0 \mathrm{keV}$ band and $\mathrm{S}$ is count rate in $0.5-2.0 \mathrm{keV}$ band. We present seven new LLAGN candidates in Table 4. LLAGN selection is performed using following indicators:

$-X / O(>-1)$

- Galaxy colour (Red)

- Hardness ratio (> -0.55)

- Total X-ray counts (>100 cts)

- X-ray luminosity $\left(>10^{41} \mathrm{erg} \mathrm{s}^{-1}\right)$

These indicators are very efficient to identify X-ray AGNs, and similar methods were applied to other AGN candidates on different surveys (e.g., Xue et al. 2011; Ranalli 2012; Vattakunnel et al. 2012; Marchesi et al. 2016). 

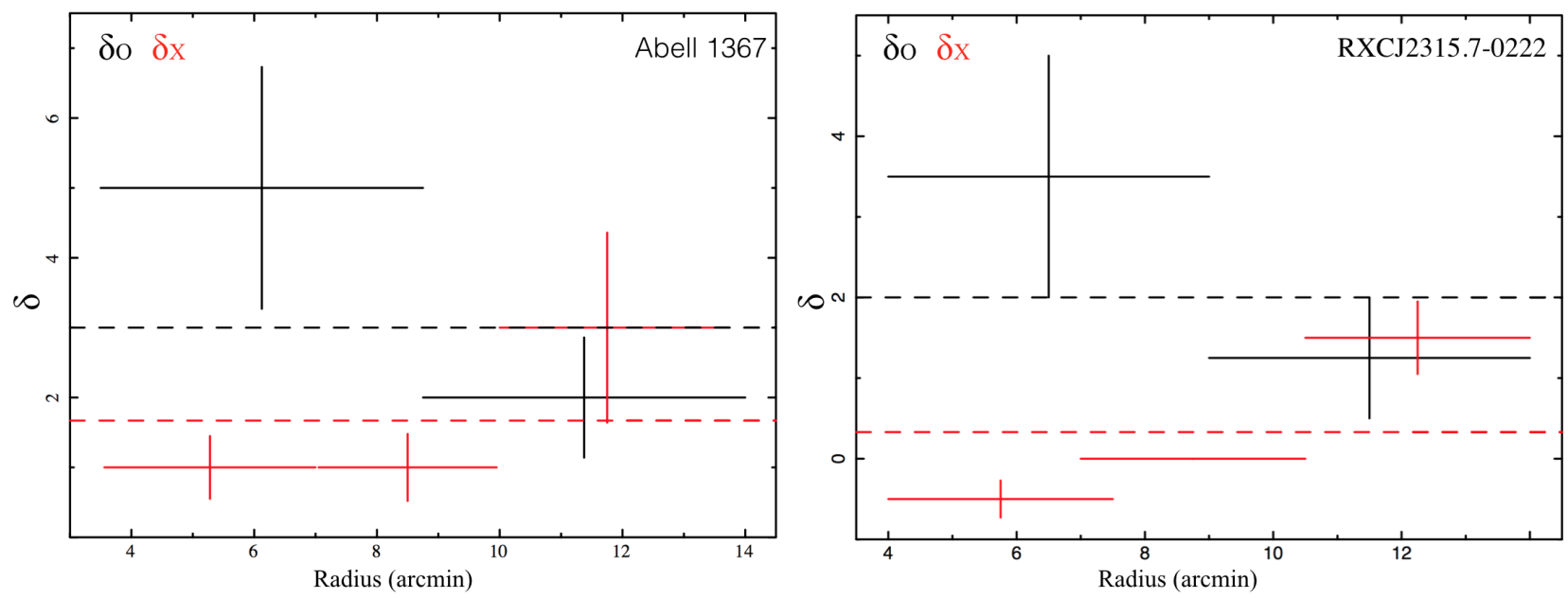

Figure 4. X-ray versus optical overdensity as a function of the distance from the centre of the cluster. The red dashed line corresponds to the mean X-ray overdensity of relative galaxy cluster, and the black dashed line represents optical overdensity of relative galaxy cluster.

Table 4. X-ray to optical properties of new LLAGN candidates.

\begin{tabular}{ccccc}
\hline \hline Source Name & HR & $\begin{array}{c}\log \left(\mathrm{L}_{X}\right) \\
\mathrm{erg} \mathrm{s}^{-1}\end{array}$ & $X / O$ & Cluster \\
\hline XMMU J140721.6-264716 & $0.62 \pm 0.11$ & 41.30 & -0.68 & A3581 \\
XMMU J113408.4+490318 & $-0.54 \pm 0.07$ & 41.24 & -0.02 & A1314 \\
XMMU J140215.6-113748 & $-0.07 \pm 0.05$ & 41.84 & 0.21 & A1836 \\
XMMU J011105.5-612548 & $0.29 \pm 0.11$ & 41.16 & -0.61 & AS137 \\
XMMU J010949.4-613153 & $-0.33 \pm 0.09$ & 41.13 & 0.02 & AS137 \\
XMMU J141216.6-342422 & $-0.45 \pm 0.04$ & 41.46 & -0.10 & AS758 \\
XMMU J141308.6-342105 & $0.30 \pm 0.22$ & 41.49 & -0.96 & AS758 \\
\hline
\end{tabular}

\section{CONCLUSIONS}

In this work, we studied ten nearby $(\leqslant 171 \mathrm{Mpc})$ galaxy clusters. Within these clusters, we detected 874 point-like sources; a fraction of them (483) is expected to be a false detection related to the diffuse ICM emission. We removed those sources located in the central regions of galaxy clusters $\left(95 \leqslant \mathrm{r}_{c} \leqslant 145 \mathrm{kpc}\right)$ from our final sample unless they are bright enough to be detected within ICM. All the pointlike sources within $0.3-10 \mathrm{keV}$ spectra were fitted with an absorbed power-law; a minority of spectra showed evidence of thermal emission lines, which we fitted adding a thermal component (APEC). We calculated the $\log N-\log S$ for our samples and we compared cluster results with those obtained in the Lockman Hole and in the Hubble Deep Field North. The number counts are a factor $\sim 2$ higher in the clusters than they are in the fields, at any flux level. In the luminosity range $\left(40.08 \leqslant \log \left(\mathrm{L}_{X}\right) \leqslant 42.39 \mathrm{erg} \mathrm{s}^{-1}\right)$ of the point-like sources in our sample, X-ray emission is mostly produced from LLAGNs, XRBs and star formation. Although starburst and normal galaxies dominate large fraction of X-ray sources of our survey, the fraction of LLAGNs is nonetheless significant. Using proxies such as $X / O, L_{X} / L_{B}$ and $L_{X} / L_{K}$, we found significant $X$-ray excess in several galaxies. By considering X-ray excess of member galaxies, we linked the nature of X-ray emission to two different processes: AGN triggering and star formation. We used efficient indicators to separate LLAGNs and star-forming galaxies. In the major- ity of the red galaxies, the enhanced X-ray emission can be explained by AGN activity; nevertheless X-ray emission can be produced by unresolved XRBs in some cases. For the blue galaxies, we explained X-ray excess with star formation, which can be related to an extreme number of HMXBs and/or contributions from SNRs. Due to the absence of redshift information of X-ray sources, we assumed all X-ray sources in our survey as cluster members, and we compared X-ray and optical overdensities of our sample of clusters. We found that X-ray overdensities are significantly lower than optical overdensities in our survey, which can be explained by the fact that X-ray sources are suppressed within cluster environments. We also note that some non-redshift X-ray sources may not be cluster members. In that case, calculated $\mathrm{X}$-ray overdensities may decrease, and suppression of X-ray sources in cluster environments even becomes clearer. The absence of very bright X-ray sources $\left(\mathrm{L}_{X}>10^{42} \mathrm{erg} \mathrm{s}^{-1}\right)$ in nearby galaxy clusters indicates that X-ray AGNs are the highly suppressed within the central regions of clusters due to highly pressurised environment. We still note that although dense and hot ICM suppress X-ray AGNs, AGN fuelling can still be effective in the sparse parts of ICM. As possible as this scenario is, we conclude that the large majority X-ray bright galaxies at the outskirts of clusters are dominated by star formation activities. Furthermore, we explain the existence of LLAGNs within clusters with close encounters of galaxies rather than AGN fuelling. Consequently, we contributed the suppression/triggering conflict in favour of the suppression by studying ten nearby galaxy clusters. However, the number of clusters in our sample is quite low, and more SDSS and XMM-Newton observations of nearby galaxy clusters are required to solve the conflict.

\section{ACKNOWLEDGEMENTS}

We are grateful to the anonymous referee for comments that significantly improved this article. We would like to thank Guenther Hasinger, Marat Gilfanov, Ho Seong Hwang, Elias Koulouridis, Piero Ranalli and Stefano Marchesi for their valuable comments and suggestions. We acknowledge the fi- 

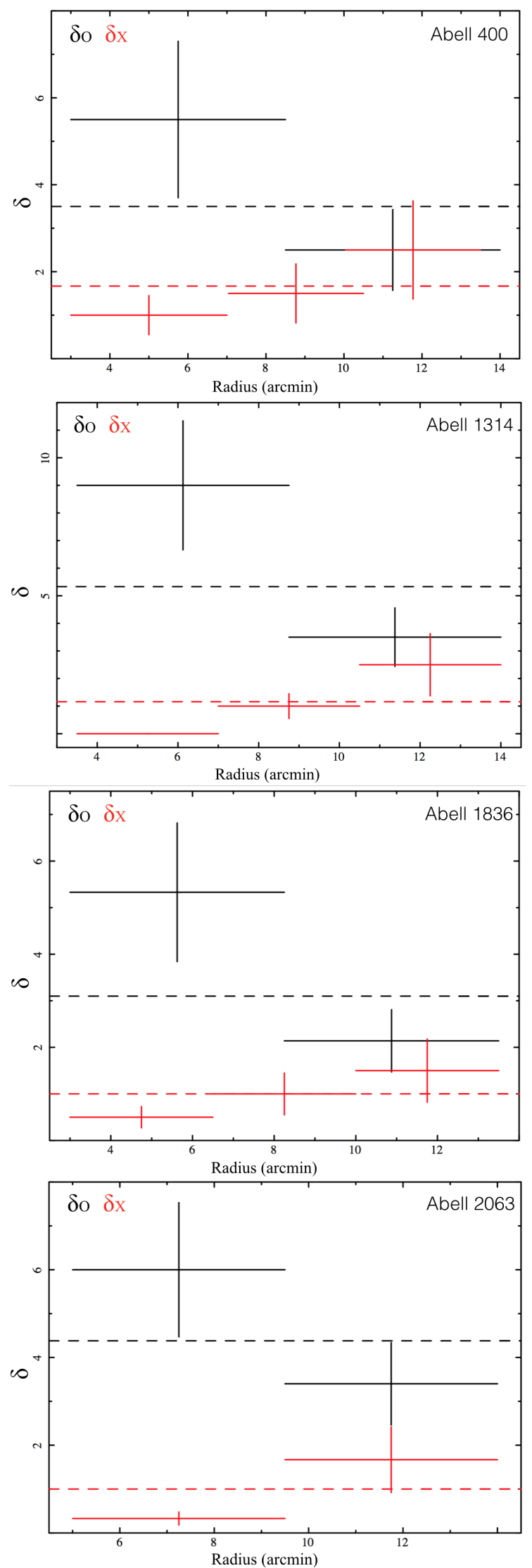

Figure 5. X-ray versus optical overdensity as a function of the distance from the centre of the cluster. The red dashed line corresponds to the mean X-ray overdensity of relative galaxy cluster, and the black dashed line represents optical overdensity of relative galaxy cluster.

MNRAS 471, 4990-5007 (2017)
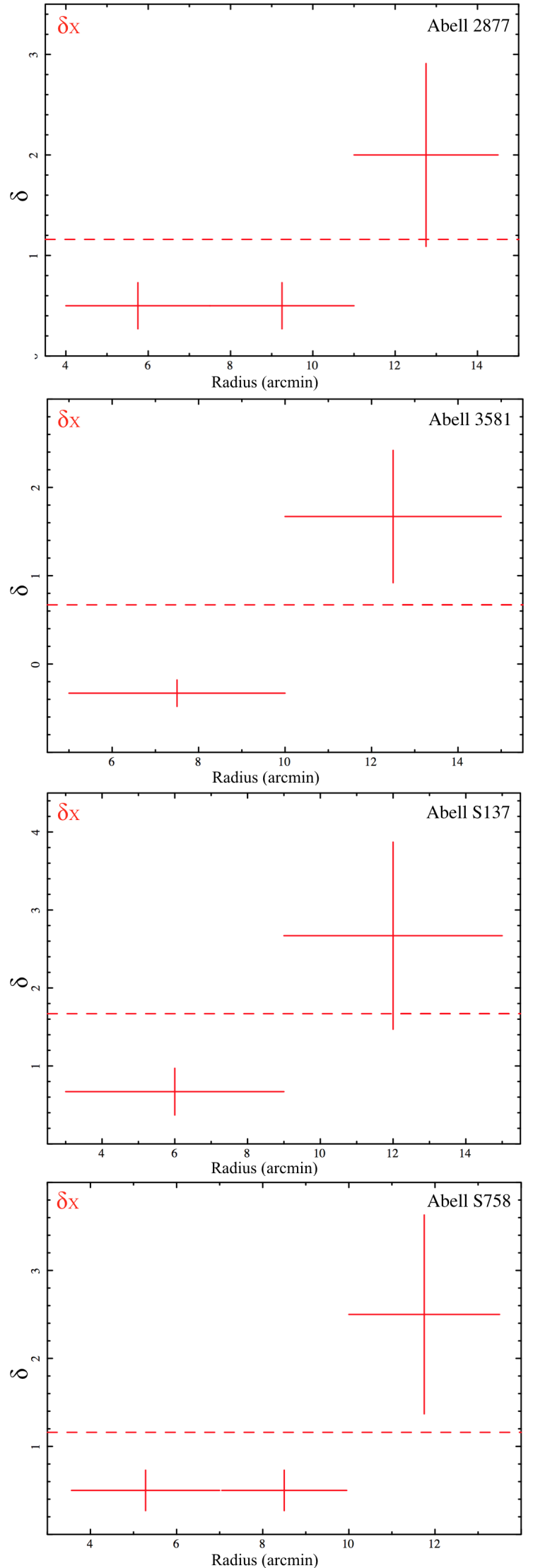

Figure 6. X-ray versus optical overdensity as a function of the distance from the centre of the cluster. The red dashed line corresponds to the mean X-ray overdensity of relative galaxy cluster, and the black dashed line represents optical overdensity of relative galaxy cluster. 
nancial support provided by The Scientific and Technological Research Council of Turkey through grant no: 113F117.

The authors also would like to thank YTU Scientific Research \& Project Office (BAP) funding with contact number 2013-01-01-KAP04. 


\section{REFERENCES}

Alberts S., et al., 2016, ApJ, 825, 72

Alexander D. M., Hickox R. C., 2012, NewAR, 56, 93

Balogh M. et al., 1997, ApJ, 488, 75

Balogh M. et al., 1999, ApJ, 527, 54

Bufanda E. et al., 2017, MNRAS, 465, 2531

Brusa M. et al., 2010, ApJ, 716, 348

Bridle A. H., Perley R. A., 1984, ARAA\&A, 22, 319

Butcher H., Oemler A., 1978, ApJ, 226, 559

Cappelluti N. et al., 2005, A\&A, 430, 39

Cappi M. et al., 2001, ApJ, 548, 624

Condon J. J., 1992, ARA\&A, 30, 575

Condon J. J. et al. 1998, AJ, 115, 1693

Cortese L., Gavazzi G., Boselli A., Iglesias-Paramo J., Carrasco L., 2004 A\&A, 425, 429

Dai X., Griffin R. D., Kochanek C. S., Nugent J. M., Bregman J. N., 2015, ApJs, 218, 8

Dalya G. et al., 2016, VizieR Online Data Catalog, 7275

D'Elia V. et al., 2004, NuPhS, 132, 54

Dressler A., 1980, ApJ, 236, 351

Dressler A. et al., 1999, ApJs, 122, 51

Ehlert S. et al., 2013, MNRAS, 428, 3509

Ehlert S. et al., 2014, MNRAS, 428, 3509

Ellis S. C., O'Sullivan E., 2006, MNRAS, 367, 627

Ellison S. L., Patton D. R., Mendel J. T., Scudder J. M., 2011, MNRAS, 418, 2043

Fabbiano G., Feigelson E., Zamorani G., 1982, ApJ, 259, 367

Fabbiano G., 2006, ARA\&A, 44, 323

Fassbender R., Šuhada R., Nastasi A., 2012, AdAst, 2012, 32

Fiore F. et al., 2003, A\&A, 409, 79

Flesch E., 2010, PASA, 27, 283

Gavazzi G., Savorgnan G., Fumagalli M., 2011, A\&A, 534, 31

Getman, K. V. et al., 2005, ApJs, 160, 319

Gilmour R., Best P., Almaini O., 2009, MNRAS, 392, 1509

Gisler, G. R. 1978, MNRAS, 183, 633

Grimm H. J., McDowell J., Zezas A., Kim D. W., Fabbiano G., 2005, ApJS, 161, 271

Haggard D. et al., 2010, ApJ, 723, 1447

Haines C. P. et al., 2012, ApJ, 754, 97

Hasinger G. et al., 2001, A\&A, 365, 45

Helfand D. J., Moran E. C., 2001, ApJ, 554, 27

Ho L. C. et al., 2001, ApJ, 549, 51

Hudaverdi M. et al., 2006, PASJ, 58, 931

Hwang H. S., Lee M. G., 2009, MNRAS, 397, 2111

Kauffmann G. et al., 2003, MNRAS, 341, 33

Kauffmann G. et al., 2004, MNRAS, 353, 713

Khabiboulline E. T. et al., 2014, ApJ, 795, 62

King I., 1962, AJ, 67, 471

Kormendy J., Richstone D., 1995, ARA\&A, 33, 581

Koulouridis E., Plionis M., 2010, ApJ, 714, 181

Koulouridis E. et al., 2014, A\&A, 567, 83

Koulouridis E. et al., 2016, A\&A, 592, 11

Laganá T. F., Dupke R. A., Sodré L. Jr., Lima Neto G. B., Durret, F., 2009, MNRAS, 394, 357

LaMassa S. M. et al., 2013, MNRAS, 436, 3581

Leaman J., Li W., Chornock R., Filippenko, A. V., 2011, MNRAS, 412,1419

Lewis I. et al., 2002, MNRAS, 334, 673

Lietzen H., et al., 2011, A\&A, 535, 21

Lin Y., Mohr J. J., 2004, ApJ, 617, 879

Loaring N. S. et al., 2013, MNRAS, 362, 1371

López-Cruz O., Barkhouse W. A., Yee H. K. C., 2004, ApJ, 614, 679

Maccacaro T. et al., 1998, ApJ, 326, 680

Marchesi S. et al, 2016, ApJ, 817, 34

Martini P., Kelson D. D., Kim E., Mulchaey J. S., Athey A. A., 2006, AJ, 644, 116
Masters K. L. et al., 2010, MNRAS, 405, 783

Matsushita K., 2001, ApJ, 547, 693

Marcum P. M. et al., 2001, ApJS, 132, 129

Melnyk O. et al., 2013, A\&A, 557, 81

Mineo S., Gilfanov M., Sunyaev R., 2012, MNRAS, 419, 2095

Montero-Dorta A. D., Prada F., 2009, MNRAS, 399, 1106

Muno M. P. et al., 2004, ApJ, 613, 1179

Muzzin A., Wilson, G., Lacy, M., Yee, H. K. C., Stanford, S. A., 2008, ApJ, 686, 966

Miller N. A., Owen F. N., 2003, AJ, 125, 2427

Neal A. M., Frazer N. O., 2003, ApJ, 125, 5

Oemler A. Jr, 1974, ApJ, 194, 1

Park S. Q. et al., 2008, APJ, 678, 744

Peres, C. B. et al., 1998, MNRAS, 298, 416

Pimbblet K. A. et al., 2002, MNRAS, 331, 333

Popesso P., Biviano A., 2006, A\&A, 460, 23

Pineau F. X. et al., 2011, A\&A, 527, 126

Poggianti B. M., 1997, A\&AS, 122, 399

Ranalli P., Comastri A., Setti G., 2003, A\&A, 399, 39

Ranalli P., Comastri A., Setti G., 2003, A\&A, 440, 23

Ranalli P. et al., 2012, A\&A, 542, 16

Ruderman, J. T., Ebeling, H., 2005, APJ, 623, 81

Schaefer A. L. et al., 2017, MNRAS, 464, 121

Schlafly E. F., Finkbeiner D. P., 2011, ApJ, 737, 103

Shirazi M., Brinchmann J., 2012, MNRAS, 421, 1043

Sun M., 2009, ApJ, 704, 1586

Thomas C. F. et al., 2008, A\&A, 486, 755

Tojeiro R. et al, 2013, MNRAS, 432, 359

Tully R. B., Mould J. R., Aaronson M., 1982, ApJ, 257, 527

Tully R. B., 2015, AJ, 2015, 149, 171

Van den Bergh S., 1976, ApJ, 206, 883

Van Velzen S. et al, 2012, A\&A, 544, 18

Vattakunnel S. et al., 2012, MNRAS, 420, 2190

de Vaucouleurs G. et al., 1991, RC3, 9, 0

Véron-Cetty M. P., Véron P., 2010, A\&A, 518, 10

Wagner C. R. et al., 2017, ApJ, 834, 53

Wetzel A. R., Tinker J. L., Conroy C., van den Bosch F. C., 2014, MNRAS, 439, 2687

Xue Y. Q. et al., 2011, ApJs, 195, 10

Zacharias N. et al., 2005, AAS, 205, 4815

Zacharias N. et al., 2013, AJ, 145, 44 
Table A1. X-ray to optical properties of individual galaxies.

\begin{tabular}{|c|c|c|c|c|c|c|c|c|c|c|}
\hline $\begin{array}{l}\text { Object Name } \\
\text { (I) }\end{array}$ & $\begin{array}{l}\text { Redshift } \\
\text { (II) }\end{array}$ & $\begin{array}{c}\mathrm{m}_{r} \\
(\mathrm{III})\end{array}$ & $\begin{array}{l}X / O \\
(\mathrm{IV})\end{array}$ & $\begin{array}{l}\mathrm{m}_{B} \\
(\mathrm{~V})\end{array}$ & $\begin{array}{l}\mathrm{m}_{K} \\
(\mathrm{VI})\end{array}$ & $\begin{array}{c}\log \mathrm{L}_{X} \\
(\mathrm{VII})\end{array}$ & $\begin{array}{c}\log \left(\mathrm{L}_{K} / \mathrm{L}_{\odot}\right) \\
(\mathrm{VIII})\end{array}$ & $\begin{array}{c}\log \left(\mathrm{L}_{B} / \mathrm{L}_{\odot}\right) \\
(\mathrm{IX})\end{array}$ & $\begin{array}{l}\text { Type } \\
\text { (X) }\end{array}$ & $\begin{array}{l}\text { Cluster } \\
\text { (XI) }\end{array}$ \\
\hline CGCG 415-040 & 0.022980 & 14.35 & -2.19 & 14.53 & 10.34 & 40.70 & 11.15 & 10.33 & So & $\mathrm{A} 400$ \\
\hline CGCG 415-046 & 0.022820 & 14.50 & -2.28 & 14.48 & 10.79 & 40.55 & 10.96 & 10.34 & $\mathrm{E}$ & $\mathrm{A} 400$ \\
\hline $3 \mathrm{C} 75 \mathrm{~A}$ & 0.022580 & 14.99 & -1.48 & 13.86 & 9.32 & 41.25 & 11.53 & 10.56 & $\mathrm{E}$ & $\mathrm{A} 400$ \\
\hline $3 \mathrm{C} 75 \mathrm{~B}$ & 0.024113 & 13.10 & -2.66 & 15.00 & 12.15 & 40.71 & 10.46 & 10.17 & So & $\mathrm{A} 400$ \\
\hline 2MASX J02574741+0601395 & 0.024811 & 15.09 & -1.93 & 15.72 & 11.29 & 40.67 & 10.83 & 9.91 & $\mathrm{E}$ & $\mathrm{A} 400$ \\
\hline 2MASX J01100662-4555544 & 0.024360 & 15.46 & -1.38 & 16.14 & 12.42 & 41.05 & 10.38 & 9.74 & So & A2877 \\
\hline 2MASX J01101993-4551184 & 0.023243 & none & none & 15.31 & 10.61 & 40.84 & 11.06 & 10.03 & So & A2877 \\
\hline IC1633 & 0.024240 & 12.95 & -2.14 & 12.40 & 8.39 & 41.85 & 11.98 & 11.23 & E1 & A2877 \\
\hline ESO 243- G 049 & 0.022395 & 14.29 & -1.77 & 14.77 & 10.70 & 41.08 & 10.99 & 10.21 & $\mathrm{Sa} 0$ & A2877 \\
\hline ESO 243- G 051 & 0.021855 & 14.00 & -2.68 & 13.62 & 10.01 & 40.36 & 11.23 & 10.64 & $\mathrm{Sb}$ & A2877 \\
\hline ESO 243- G 045 & 0.025881 & 13.81 & -2.25 & 13.32 & 9.71 & 40.87 & 11.51 & 10.92 & So & A2877 \\
\hline NGC 3851 & 0.021130 & 14.41 & -2.29 & 15.12 & 11.01 & 40.48 & 10.89 & 10.10 & $\mathrm{E}$ & A1367 \\
\hline NGC 3860 & 0.018663 & 14.32 & -1.64 & 13.77 & 10.39 & 41.17 & 10.99 & 10.49 & $\mathrm{Sa}$ & A1367 \\
\hline NGC $3860 \mathrm{~B}$ & 0.028250 & 15.69 & -1.91 & 14.99 & 13.35 & 40.35 & 10.17 & 10.37 & $\mathrm{~S}$ & A1367 \\
\hline NGC 3861 & 0.016900 & 13.88 & $\begin{array}{l}-2.42 \\
-2.42\end{array}$ & 12.93 & 9.95 & 40.56 & 11.12 & 10.78 & $\mathrm{~S}$ & A1367 \\
\hline NGC 3862 & 0.021718 & 13.64 & -1.37 & 13.51 & 9.48 & 41.76 & 11.52 & 10.76 & $\mathrm{E}$ & A1367 \\
\hline CGCG 097-125 & 0.027436 & 15.06 & -1.77 & 15.23 & 11.46 & 40.74 & 10.92 & 10.27 & $\mathrm{E}$ & A1367 \\
\hline NGC 3842 & 0.021068 & 12.18 & -3.12 & 12.62 & 9.07 & 40.55 & 11.65 & 11.09 & $\mathrm{E}$ & A1367 \\
\hline GALEXASC J114359.29+195633.6 & 0.023323 & 19.74 & -0.08 & 21.38 & none & 40.56 & none & 7.67 & & A1367 \\
\hline 2MASX J15225650+0839004 & 0.03361 & 15.00 & -2.09 & 15.84 & 11.91 & 40.86 & 10.90 & 10.18 & So & A2063 \\
\hline CGCG 077-097 & 0.034174 & 13.14 & -1.96 & 14.36 & 10.07 & 41.72 & 11.65 & 10.79 & $\mathrm{~S} ?$ & A2063 \\
\hline 2MASX J15231224+0832590 & 0.036619 & 15.20 & -0.49 & 15.20 & 12.41 & 42.39 & 10.78 & 10.51 & $\mathrm{Sa}$ & A2063 \\
\hline MCG -02-36-002 & 0.037776 & 12.74 & -2.61 & 14.10 & 9.97 & 41.31 & 11.79 & 10.99 & $\mathrm{Sa} 0$ & A1836 \\
\hline 2MASX J14015570-1138043 & 0.036979 & 16.57 & -1.79 & 18.15 & 14.09 & 40.59 & 10.12 & 9.35 & & A1836 \\
\hline 2MASX J14013206-1139261 & 0.041662 & 15.55 & -1.74 & 15.60 & 11.86 & 41.12 & 11.12 & 10.47 & & A1836 \\
\hline IC 708 & 0.031679 & 13.07 & -2.54 & 13.85 & 10.09 & 41.09 & 11.58 & 10.93 & $\mathrm{E}$ & A1314 \\
\hline IC 711 & 0.032436 & 13.88 & -2.59 & 14.88 & 11.08 & 40.72 & 11.21 & 10.54 & $\mathrm{E} ?$ & A1314 \\
\hline IC 712 & 0.033553 & 13.13 & -2.98 & 14.05 & 9.89 & 40.68 & 11.71 & 10.90 & $\mathrm{~S} ?$ & A1314 \\
\hline 2MASX J11340896+4915162 & 0.037230 & 16.25 & -0.22 & 16.99 & 13.47 & 42.28 & 10.33 & 9.78 & & A1314 \\
\hline $\mathrm{MCG}+08-21-065$ & 0.029670 & 15.14 & -0.58 & 15.21 & 11.88 & 42.15 & 10.77 & 10.29 & $\mathrm{Sb}$ & A1314 \\
\hline LEDA 97398 & 0.031600 & 19.70 & -0.38 & 20.80 & none & 40.65 & none & 8.11 & & A1314 \\
\hline IC 4374 & 0.021798 & 13.79 & -1.44 & 15.24 & 9.54 & 41.58 & 11.49 & 10.06 & $\mathrm{Sa} 0$ & A3581 \\
\hline ESO 510- G 065 & 0.025671 & 14.21 & -2.76 & 16.11 & 11.90 & 40.24 & 10.68 & 9.85 & $\mathrm{Sb}$ & A 3581 \\
\hline ESO 510- G 066 & 0.024333 & 13.78 & -1.88 & 15.13 & 9.92 & 41.24 & 11.43 & 10.19 & $\mathrm{Sa} 0$ & A3581 \\
\hline MCG-06-31-029 & 0.038500 & 11.10 & -3.89 & 11.20 & 11.15 & 40.72 & 11.34 & 12.17 & $\mathrm{E}+$ & AS758 \\
\hline 2MASX J14122917-3417417 & 0.043003 & 12.41 & -3.49 & 15.36 & 11.24 & 40.67 & 11.39 & 10.60 & & AS758 \\
\hline NGC 0432 & 0.026929 & 13.82 & -2.89 & 13.92 & 9.93 & 40.29 & 11.47 & 10.72 & So & AS137 \\
\hline 2MASX J01125179-6139513 & 0.026442 & 14.05 & -2.65 & 14.75 & 10.70 & 40.43 & 11.15 & 10.38 & $\mathrm{E}$ & AS137 \\
\hline NGC 7556 & 0.025041 & 12.21 & -3.23 & 15.39 & 9.24 & 40.56 & 11.65 & 10.04 & So & RXCJ2315.7-0222 \\
\hline NGC 7566 & 0.026548 & 13.03 & -3.12 & 13.66 & 10.19 & 40.32 & 11.33 & 10.79 & $\mathrm{Sb} ?$ & RXCJ2315.7-0222 \\
\hline
\end{tabular}

\section{APPENDIX A: X-RAY TO OPTICAL PROPERTIES OF GALAXIES}

X-ray and optical properties of identified member galaxies I) Galaxy names II) Redshift values from Ned Astronomical Database III) R-band magnitude values from Vizier database IV) X-ray to optical flux ratio V) Apparent blue magnitude values from Vizier database VI) Apparent k magnitude values are taken from Tully (2015); Dalya et al. (2016) VII) Hard band logarithmic X-ray luminosity values from spectral analysis VIII) K-band luminosities calculated from extinction corrected k-band magnitude. IX) Logarithmic blue optical luminosity values calculated from extinction corrected b-band magnitudes X) Morphological type of galaxies XI) Name of cluster hosts identified galaxies. 
Table B1. Galaxy Colour Survey

\begin{tabular}{|c|c|c|c|c|c|c|c|c|c|}
\hline Object Name & HR & $\begin{array}{c}\log f_{X} \\
\operatorname{erg~cm^{-2}} \mathrm{s}^{-1} \\
\text { III }\end{array}$ & $\begin{array}{c}\log \mathrm{L}_{X} \\
\operatorname{erg~s}{ }^{-1} \\
\text { IV }\end{array}$ & $\begin{array}{c}\mathrm{r} \\
\mathrm{mag} \\
\mathrm{V}\end{array}$ & $\begin{array}{c}\text { g-r } \\
\text { mag } \\
\text { VI }\end{array}$ & $\begin{array}{c}\mathrm{d} \\
\mathrm{Mpc} \\
\mathrm{VII}\end{array}$ & $\begin{array}{c}\mathrm{P} \\
\% \\
\text { VIII }\end{array}$ & Type & Cluster \\
\hline XMMU J025824.6+060248 & $-0.61 \pm 0.05$ & -12.72 & 41.40 & 18.58 & 0.33 & 0.31 & 84 & Blue & A400 \\
\hline XMMU J025749.2+055136 & $-0.49 \pm 0.09$ & -12.95 & 41.18 & 21.09 & 0.02 & 0.29 & 98 & Blue & $\mathrm{A} 400$ \\
\hline XMMU J025747.3+060942 & $-0.54 \pm 0.13$ & -13.43 & 40.70 & 20.67 & -0.04 & 0.24 & 91 & Blue & A400 \\
\hline XMMU J025718.5+060022 & $-0.57 \pm 0.15$ & -13.34 & 40.76 & 20.73 & 0.59 & 0.17 & 92 & Blue & A400 \\
\hline XMMU J025730.8+060545 & $-0.34 \pm 0.20$ & -13.44 & 40.69 & 18.53 & 0.64 & 0.15 & 97 & Blue & $\mathrm{A} 400$ \\
\hline XMMU J025718.8+060813 & $-0.45 \pm 0.21$ & -13.48 & 40.65 & 17.60 & 0.84 & 0.25 & 96 & Red & $\mathrm{A} 400$ \\
\hline XMMU J025806.2+055327 & $0.09 \pm 0.32$ & -13.31 & 40.82 & 20.91 & 0.02 & 0.29 & 95 & Red & A400 \\
\hline XMMU J025712.6+055960 & $0.88 \pm 0.17$ & -13.42 & 40.71 & 21.07 & 0.66 & 0.21 & 96 & Red & $\mathrm{A} 400$ \\
\hline XMMU J113421.6+490050 & $-0.60 \pm 0.08$ & -13.47 & 40.95 & 19.47 & 0.66 & 0.24 & 98 & Blue & A1314 \\
\hline XMMU J113408.4+490318 & $-0.54 \pm 0.11$ & -13.16 & 41.24 & 19.09 & 1.01 & 0.28 & 83 & Red & A1314 \\
\hline XMMU J113548.5+491150 & $-0.44 \pm 0.24$ & -13.48 & 40.93 & 20.95 & 0.48 & 0.49 & 95 & Blue & A1314 \\
\hline XMMU J114435.0+195131 & $-0.58 \pm 0.08$ & -13.07 & 40.97 & 20.01 & 0.65 & 0.20 & 94 & Blue & A1367 \\
\hline XMMU J114427.1+194338 & $-0.62 \pm 0.18$ & -13.32 & 40.72 & 21.09 & 0.45 & 0.09 & 89 & Blue & A1367 \\
\hline XMMU J114452.5+195133 & $-0.55 \pm 0.10$ & -13.02 & 41.02 & 21.89 & 0.32 & 0.21 & 98 & Blue & A1367 \\
\hline XMMU J114515.8+194951 & $-0.61 \pm 0.28$ & -13.38 & 40.66 & 19.84 & 0.27 & 0.28 & 97 & Blue & A1367 \\
\hline XMMU J114436.5+195336 & $-0.29 \pm 0.30$ & -13.48 & 40.56 & 20.89 & 0.19 & 0.25 & 99 & Blue & A1367 \\
\hline XMMU J114359.3+195632 & $-0.73 \pm 0.21$ & -13.48 & 40.56 & 21.75 & 0.70 & 0.30 & 73 & Red & A1367 \\
\hline XMMU J114507.7+193552 & $-0.82 \pm 0.37$ & -13.49 & 40.55 & 21.27 & 1.09 & 0.31 & 92 & Red & A1367 \\
\hline XMMU J140215.6-113748 & $-0.07 \pm 0.10$ & -12.63 & 41.84 & 18.36 & 1.05 & 0.37 & 96 & Red & A1836 \\
\hline XMMU J140207.9-113553 & $-0.63 \pm 0.08$ & -13.42 & 41.06 & 19.53 & 0.11 & 0.28 & 94 & Blue & A1836 \\
\hline XMMU J140135.5-112708 & $-0.15 \pm 0.34$ & -13.39 & 41.05 & 19.28 & 1.51 & 0.42 & 93 & Red & A1836 \\
\hline XMMU J140043.8-113731 & $-0.29 \pm 0.40$ & -13.42 & 41.18 & 17.92 & 1.30 & 0.63 & 96 & Red & A1836 \\
\hline XMMU J152342.5+084535 & $-0.82 \pm 0.32$ & -13.22 & 41.23 & 22.00 & 0.96 & 0.55 & 74 & Red & A2063 \\
\hline XMMU J152250.9+084447 & $0.41 \pm 0.76$ & -13.21 & 41.21 & 21.34 & 1.15 & 0.38 & 92 & Red & A2063 \\
\hline XMMU J152322.3+082159 & $-0.20 \pm 0.48$ & -13.22 & 41.23 & 19.99 & 0.23 & 0.64 & 97 & Blue & A2063 \\
\hline XMMU J231638.6-022527 & $-0.56 \pm 0.07$ & -12.54 & 41.67 & 19.05 & 0.20 & 0.42 & 99 & Blue & RXCJ2315.7-0222 \\
\hline XMMU J231624.5-021457 & $-0.77 \pm 0.07$ & -13.35 & 40.80 & 20.63 & 1.57 & 0.40 & 81 & Red & RXCJ2315.7-0222 \\
\hline XMMU J231555.9-021644 & $0.33 \pm 0.29$ & -13.33 & 40.81 & 23.84 & 0.44 & 0.21 & 93 & Blue & RXCJ2315.7-0222 \\
\hline XMMU J231610.3-021502 & $-0.64 \pm 0.31$ & -13.47 & 40.67 & 20.19 & 1.43 & 0.31 & 91 & Red & RXCJ2315.7-0222 \\
\hline XMMU J231500.7-022515 & $-0.25 \pm 0.27$ & -13.36 & 40.85 & 18.72 & 0.72 & 0.34 & 97 & Blue & RXCJ2315.7-0222 \\
\hline Object Name & $\mathrm{HR}$ & $\begin{array}{c}\log f_{X} \\
\operatorname{erg~cm^{-2}} \mathrm{s}^{-1}\end{array}$ & $\begin{array}{c}\log \mathrm{L}_{X} \\
\operatorname{erg~s}{ }^{-1}\end{array}$ & $\begin{array}{c}\mathrm{R} \\
\mathrm{mag}\end{array}$ & $\begin{array}{l}\text { B-R } \\
\text { mag }\end{array}$ & $\begin{array}{c}\mathrm{d} \\
\mathrm{Mpc}\end{array}$ & $\begin{array}{l}\mathrm{P} \\
\%\end{array}$ & Type & Cluster \\
\hline I & II & III & IV & V & VI & VII & VIII & IX & $\mathrm{X}$ \\
\hline XMMU J010914.4-455914 & $-0.61 \pm 0.09$ & -13.02 & 41.13 & 18.98 & 0.74 & 0.23 & 93 & Blue & A2877 \\
\hline XMMU J010952.4-460536 & $-0.63 \pm 0.26$ & -13.47 & 40.67 & 19.37 & 0.59 & 0.29 & 96 & Blue & A 2877 \\
\hline XMMU J011024.5-454426 & $-0.82 \pm 0.27$ & -13.44 & 40.70 & 18.97 & 0.48 & 0.36 & 98 & Blue & A 2877 \\
\hline XMMU J010858.9-455136 & $-0.01 \pm 0.49$ & -13.20 & 40.93 & 20.18 & 1.09 & 0.31 & 99 & Red & A 2877 \\
\hline XMMU J140721.6-264716 & $0.62 \pm 0.16$ & -12.77 & 41.30 & 16.47 & 1.65 & 0.40 & 92 & Red & A3581 \\
\hline XMMU J140803.6-270841 & $-0.21 \pm 0.60$ & -13.49 & 40.58 & 20.17 & 1.31 & 0.31 & 85 & Red & A3581 \\
\hline XMMU J140825.4-270849 & $-0.39 \pm 0.35$ & -13.25 & 40.82 & 20.17 & 0.71 & 0.42 & 85 & Blue & A3581 \\
\hline XMMU J011205.4-613255 & $-0.56 \pm 0.09$ & -13.47 & 40.73 & 20.16 & 0.77 & 0.08 & 99 & Blue & AS137 \\
\hline XMMU J011127.4-612612 & $-0.39 \pm 0.24$ & -13.49 & 40.70 & 18.35 & 0.87 & 0.18 & 99 & Blue & AS137 \\
\hline XMMU J011105.5-612548 & $0.29 \pm 0.20$ & -13.02 & 41.16 & 17.28 & 1.63 & 0.24 & 88 & Red & AS137 \\
\hline XMMU J010949.4-613153 & $-0.33 \pm 0.16$ & -13.06 & 41.13 & 18.95 & 1.57 & 0.44 & 62 & Red & AS137 \\
\hline XMMU J011213.1-612015 & $-0.43 \pm 0.20$ & -13.36 & 40.84 & 19.34 & 0.49 & 0.37 & 99 & Blue & AS137 \\
\hline XMMU J141117.5-341116 & $-0.58 \pm 0.16$ & -13.09 & 41.42 & 18.65 & 0.70 & 0.74 & 87 & Blue & AS758 \\
\hline XMMU J141216.6-342422 & $-0.45 \pm 0.06$ & -13.06 & 41.46 & 18.65 & 1.70 & 0.19 & 93 & Red & AS758 \\
\hline XMMU J141308.6-342105 & $0.30 \pm 0.22$ & -13.01 & 41.49 & 16.37 & 1.58 & 0.46 & 93 & Red & AS758 \\
\hline XMMU J141245.4-342343 & $-0.46 \pm 0.10$ & -13.35 & 41.18 & 18.45 & 0.82 & 0.28 & 87 & Blue & AS758 \\
\hline XMMU J141223.0-341330 & $-0.51 \pm 0.13$ & -13.47 & 41.06 & 18.65 & 0.50 & 0.32 & 88 & Blue & AS758 \\
\hline
\end{tabular}

\section{APPENDIX B: GALAXY COLOUR - X-RAY POINT-LIKE EMISSION RELATION}

I) Source name II) Hardness Ratio [defined as (H-S)/(H+S), where $\mathrm{H}$ is count rate in $2.0-10.0 \mathrm{keV}$ and $\mathrm{S}$ is count rate in 0.5-2.0 keV] III) Logarithmic X-ray flux IV) Logarithmic Xray luminosity V) R-band magnitude VI) G-R/B-R Values VII) Projected distance from centre of related galaxy cluster VIII) Likelihood of optical association IX) Galaxy type in colour X) Cluster name. 
Table B2. Properties of point sources studied in this work.

\begin{tabular}{|c|c|c|c|c|c|c|}
\hline Object Name & Net Counts & $\begin{array}{l}\text { Log Flux } \\
\mathrm{erg} \mathrm{cm}^{-2} \mathrm{~s}^{-1}\end{array}$ & $\begin{array}{l}\text { Log Luminosity } \\
\operatorname{erg~s}^{-1}\end{array}$ & Cluster & Optical Counterpart & Redshift \\
\hline XMMU J025824.6+060248 & 750 & -12.72 & 41.40 & A400 & 2XMM J025824.6+060248 & \\
\hline XMMU J025741.5+060136 & 570 & -12.98 & 41.25 & A400 & $3 \mathrm{C} 75 \mathrm{~A}$ & 0.02258 \\
\hline XMMU J025741.8+060120 & 343 & -13.40 & 40.71 & A400 & $3 \mathrm{C} 75 \mathrm{~B}$ & 0.024113 \\
\hline XMMU J025749.2+055136 & 431 & -12.95 & 41.18 & A400 & 2XMM J025749.2+055136 & \\
\hline XMMU J025724.7+060156 & 481 & -13.21 & 40.88 & A400 & 2XMM J025724.7+060156 & \\
\hline XMMU J025808.1+055808 & 233 & -13.34 & 40.76 & A400 & & \\
\hline XMMU J025747.3+060942 & 245 & -13.43 & 40.70 & A400 & & \\
\hline XMMU J025802.5+055448 & 221 & -13.47 & 40.67 & A400 & & \\
\hline XMMU J025802.3+055213 & 168 & -13.67 & 40.47 & A400 & & \\
\hline XMMU J025718.5+060022 & 258 & -13.34 & 40.76 & A400 & & \\
\hline XMMU J025736.7+060822 & 190 & -13.58 & 40.55 & A400 & & \\
\hline XMMU J025730.8+060545 & 197 & -13.44 & 40.69 & A400 & & \\
\hline XMMU J025718.8+060813 & 131 & -13.48 & 40.65 & A400 & & \\
\hline XMMU J025803.6+061107 & 102 & -13.49 & 40.64 & A400 & & \\
\hline XMMU J025806.2+055327 & 93 & -13.31 & 40.82 & A400 & & \\
\hline XMMU J025820.9+060008 & 107 & -13.65 & 40.48 & A400 & & \\
\hline XMMU J025747.1+060136 & 41 & -13.47 & 40.67 & A400 & 2MASX J02574741+0601395 & 0.024811 \\
\hline XMMU J025712.6+055960 & 132 & -13.42 & 40.71 & A400 & & \\
\hline XMMU J025733.7+055835 & 244 & -13.43 & 40.70 & A400 & CGCG 415-040 & 0.02298 \\
\hline XMMU J025716.6+055736 & 138 & -13.66 & 40.47 & A400 & & \\
\hline XMMU J025821.0+060537 & 120 & -13.58 & 40.55 & A400 & CGCG 415-046 & 0.02282 \\
\hline XMMU J025752.1+060631 & 97 & -13.52 & 40.61 & A400 & & \\
\hline XMMU J025810.2+055948 & 148 & -13.51 & 40.62 & A400 & & \\
\hline XMMU J025812.7+055828 & 83 & -13.92 & 40.21 & A400 & & \\
\hline XMMU J025811.2+055227 & 78 & -13.39 & 40.74 & A400 & & \\
\hline XMMU J025824.2+055810 & 58 & -13.84 & 40.29 & A400 & & \\
\hline XMMU J025702.5+060945 & 63 & -13.85 & 40.28 & A400 & & \\
\hline XMMU J025709.9+060320 & 69 & -13.70 & 40.42 & A400 & & \\
\hline XMMU J025711.7+060160 & 92 & -13.77 & 40.36 & A400 & & \\
\hline XMMU J025751.7+054843 & 49 & -13.35 & 40.76 & A400 & & \\
\hline XMMU J025712.7+061114 & 60 & -13.81 & 40.32 & A400 & & \\
\hline XMMU J025807.0+060155 & 74 & -13.77 & 40.36 & A400 & & \\
\hline XMMU J025801.6+060148 & 67 & -13.84 & 40.29 & A400 & & \\
\hline XMMU J010955.6-455551 & 1860 & -12.82 & 41.85 & A 2877 & IC1633 & 0.024240 \\
\hline XMMU J011050.6-460013 & 1245 & -13.06 & 41.09 & A2877 & GALEX J011050.4-460013.8 & \\
\hline XMMU J010914.4-455914 & 443 & -13.02 & 41.13 & A 2877 & 2XMM J010914.4-455914 & \\
\hline XMMU J011028.2-460422 & 401 & -12.99 & 41.08 & A 2877 & ESO 243- G 049 & 0.022395 \\
\hline XMMU J011119.3-455554 & 375 & -13.78 & 40.36 & A2877 & ESO 243- G 051 & 0.021855 \\
\hline XMMU J011019.9-455120 & 90 & -13.29 & 40.84 & A 2877 & 2MASX J01101993-4551184 & 0.023243 \\
\hline XMMU J010904.3-454627 & 97 & -13.27 & 40.87 & A 2877 & ESO 243- G 045 & 0.025881 \\
\hline XMMU J011007.5-455554 & 258 & -13.07 & 41.05 & A2877 & 2MASX J01100662-4555544 & 0.024360 \\
\hline XMMU J010942.5-455357 & 49 & -13.90 & 40.24 & A 2877 & & \\
\hline XMMU J011017.7-460404 & 108 & -13.32 & 40.81 & A 2877 & & \\
\hline XMMU J010949.1-460235 & 125 & -13.72 & 40.42 & A 2877 & & \\
\hline XMMU J010952.4-460536 & 130 & -13.47 & 40.67 & A2877 & & \\
\hline XMMU J010916.4-454830 & 65 & -13.58 & 40.56 & A2877 & & \\
\hline XMMU J011032.0-455337 & 178 & -13.47 & 40.67 & A 2877 & & \\
\hline XMMU J011024.5-454426 & 102 & -13.44 & 40.70 & A 2877 & & \\
\hline XMMU J010849.6-455622 & 91 & -13.86 & 40.29 & A 2877 & & \\
\hline XMMU J010930.7-460307 & 66 & -13.65 & 40.47 & A 2877 & & \\
\hline XMMU J010947.3-455327 & 98 & -13.68 & 40.46 & A 2877 & & \\
\hline XMMU J011026.6-455246 & 72 & -13.60 & 40.54 & A 2877 & & \\
\hline XMMU J010933.6-460400 & 42 & -13.84 & 40.30 & A 2877 & & \\
\hline XMMU J010935.8-460621 & 50 & -13.46 & 40.68 & A2877 & & \\
\hline XMMU J011022.6-460105 & 86 & -13.67 & 40.47 & A2877 & & \\
\hline XMMU J010853.8-455850 & 54 & -13.77 & 40.37 & A2877 & & \\
\hline XMMU J011034.9-455149 & 100 & -13.59 & 40.55 & A 2877 & & \\
\hline XMMU J011008.8-455630 & 149 & -13.37 & 40.76 & A 2877 & & \\
\hline XMMU J011058.2-455318 & 70 & -13.68 & 40.46 & A2877 & & \\
\hline XMMU J011001.9-454917 & 78 & -13.62 & 40.52 & A 2877 & & \\
\hline XMMU J011043.2-460419 & 103 & -13.58 & 40.56 & A 2877 & & \\
\hline XMMU J010937.2-454323 & 42 & -13.61 & 40.53 & A 2877 & & \\
\hline XMMU J010858.9-455136 & 104 & -13.20 & 40.93 & A 2877 & & \\
\hline XMMU J011001.7-460818 & 49 & -13.75 & 40.39 & A2877 & & \\
\hline XMMU J011001.9-460335 & 63 & -13.97 & 40.17 & A 2877 & & \\
\hline XMMU J011006.8-455234 & 40 & -13.53 & 40.61 & A 2877 & & \\
\hline XMMU J011038.1-455829 & 41 & -13.51 & 40.62 & A 2877 & & \\
\hline XMMU J010916.1-455149 & 44 & -13.51 & 40.63 & A 2877 & & \\
\hline
\end{tabular}


Table B3. Properties of point sources studied in this work.

\begin{tabular}{|c|c|c|c|c|c|c|}
\hline Object Name & Net Counts & $\begin{array}{l}\log \text { Flux } \\
\mathrm{erg} \mathrm{cm}^{-2} \mathrm{~s}^{-1}\end{array}$ & $\begin{array}{l}\text { Log Luminosity } \\
\operatorname{erg~s}^{-1}\end{array}$ & Cluster & Optical Counterpart & Redshift \\
\hline XMMU J114505.0+193622 & 3182 & -12.28 & 41.76 & A1367 & NGC 3862 & 0.021718 \\
\hline XMMU J114435.0+195131 & 628 & -13.07 & 40.97 & A1367 & & \\
\hline XMMU J114409.4+195009 & 576 & -13.24 & 40.81 & A1367 & & \\
\hline XMMU J114448.9+194742 & 454 & -12.86 & 41.17 & A1367 & NGC 3860 & 0.018663 \\
\hline XMMU J114427.1+194338 & 303 & -13.32 & 40.72 & A1367 & & \\
\hline XMMU J114452.5+195133 & 515 & -13.02 & 41.02 & A1367 & & \\
\hline XMMU J114515.8+194951 & 136 & -13.38 & 40.66 & A1367 & & \\
\hline XMMU J114435.5+195029 & 146 & -13.52 & 40.52 & A1367 & & \\
\hline XMMU J114503.8+195826 & 103 & -13.48 & 40.56 & A1367 & NGC 3861 & $0.01690 a ̆$ \\
\hline XMMU J114436.5+195336 & 187 & -13.48 & 40.56 & A1367 & & \\
\hline XMMU J114454.7+194634 & 175 & -13.30 & 40.74 & A1367 & CGCG 097-125 & 0.027436 \\
\hline XMMU J114457.6+195302 & 145 & -13.59 & 40.45 & A1367 & & \\
\hline XMMU J114436.5+193831 & 117 & -13.52 & 40.52 & A1367 & & \\
\hline XMMU J114410.7+195327 & 100 & -13.57 & 40.47 & A1367 & & \\
\hline XMMU J114359.2+193955 & 46 & -13.42 & 40.61 & A1367 & & \\
\hline XMMU J114439.1+194525 & 167 & -13.43 & 40.61 & A1367 & & \\
\hline XMMU J114416.8+194417 & 44 & -13.48 & 40.56 & A1367 & & \\
\hline XMMU J114420.1+195849 & 41 & -13.56 & 40.48 & A1367 & & \\
\hline XMMU J114438.1+194405 & 153 & -13.45 & 40.59 & A1367 & & \\
\hline XMMU J114459.7+194742 & 96 & -13.62 & 40.41 & A1367 & & \\
\hline XMMU J114422.1+193937 & 76 & -13.46 & 40.58 & A1367 & & \\
\hline XMMU J114526.6+194345 & 43 & -13.55 & 40.49 & A1367 & & \\
\hline XMMU J114420.9+195508 & 53 & -13.71 & 40.32 & A1367 & & \\
\hline XMMU J114507.7+193552 & 74 & -13.49 & 40.55 & A1367 & & \\
\hline XMMU J114447.3+194621 & 80 & -13.69 & 40.35 & A1367 & NGC 3860B & 0.02825 \\
\hline XMMU J114501.7+194549 & 93 & -13.91 & 40.13 & A1367 & & \\
\hline XMMU J114536.9+195304 & 42 & -13.84 & 40.20 & A1367 & & \\
\hline XMMU J114537.7+195330 & 54 & -13.57 & 40.46 & A1367 & & \\
\hline XMMU J114507.7+195419 & 40 & -13.54 & 40.50 & A1367 & & \\
\hline XMMU J114507.7+195757 & 39 & -13.37 & 40.66 & A1367 & & \\
\hline XMMU J114508.4+194905 & 38 & -13.57 & 40.46 & A1367 & & \\
\hline XMMU J114402.2+195700 & 153 & -13.50 & 40.55 & A1367 & NGC 3842 & 0.021068 \\
\hline XMMU J114359.3+195632 & 59 & -13.48 & 40.56 & A1367 & GALEXASC J114359.29+195633.6 & 0.023323 \\
\hline XMMU J140729.8-270104 & 128 & -12.46 & 41.58 & A3581 & IC 4374 & 0.021798 \\
\hline XMMU J140714.2-270027 & 289 & -13.63 & 40.45 & A3581 & & \\
\hline XMMU J140715.6-270932 & 1947 & -12.89 & 41.24 & A3581 & ESO 510- G 066 & 0.024333 \\
\hline XMMU J140827.1-265828 & 106 & -13.36 & 40.72 & A3581 & & \\
\hline XMMU J140656.6-265158 & 86 & -13.79 & 40.29 & A3581 & & \\
\hline XMMU J140819.2-270150 & 109 & -13.55 & 40.52 & A3581 & & \\
\hline XMMU J140751.8-265827 & 114 & -13.97 & 40.11 & A3581 & & \\
\hline XMMU J140750.9-271138 & 130 & -13.86 & 40.22 & A3581 & & \\
\hline XMMU J140701.2-265554 & 104 & -13.87 & 40.21 & A3581 & & \\
\hline XMMU J140758.8-270425 & 91 & -13.84 & 40.24 & A3581 & & \\
\hline XMMU J140751.4-271317 & 73 & -13.63 & 40.45 & A3581 & & \\
\hline XMMU J140654.2-265314 & 56 & -13.93 & 40.15 & A3581 & & \\
\hline XMMU J140737.0-270700 & 52 & -13.99 & 40.09 & A3581 & & \\
\hline XMMU J140646.1-270112 & 69 & -13.82 & 40.25 & A3581 & & \\
\hline XMMU J140743.0-265838 & 168 & -14.00 & 40.08 & A3581 & & \\
\hline XMMU J140649.2-270031 & 51 & -13.98 & 40.09 & A3581 & & \\
\hline XMMU J140720.9-265151 & 73 & -13.58 & 40.49 & A3581 & & \\
\hline XMMU J140754.2-270555 & 118 & -13.98 & 40.09 & A3581 & & \\
\hline XMMU J140805.5-270617 & 83 & -13.78 & 40.30 & A3581 & & \\
\hline XMMU J140721.6-264716 & 134 & -12.77 & 41.30 & A3581 & & \\
\hline XMMU J140720.6-270709 & 103 & -13.68 & 40.39 & A3581 & & \\
\hline XMMU J140659.5-265213 & 58 & -13.70 & 40.37 & A3581 & & \\
\hline XMMU J140803.6-270841 & 54 & -13.49 & 40.58 & A3581 & & \\
\hline XMMU J140721.6-265328 & 50 & -13.78 & 40.29 & A3581 & & \\
\hline XMMU J140643.9-265710 & 51 & -13.87 & 40.20 & A3581 & & \\
\hline XMMU J140705.8-271047 & 71 & -13.92 & 40.16 & A3581 & & \\
\hline XMMU J140811.5-265911 & 49 & -13.83 & 40.24 & A3581 & & \\
\hline XMMU J140718.7-265415 & 97 & -13.66 & 40.42 & A3581 & & \\
\hline XMMU J140825.4-270849 & 85 & -13.25 & 40.82 & A3581 & & \\
\hline XMMU J140743.9-270648 & 102 & -13.78 & 40.29 & A3581 & & \\
\hline XMMU J140759.8-271442 & 50 & -13.29 & 40.78 & A3581 & & \\
\hline XMMU J140818.7-270533 & 46 & -13.75 & 40.33 & A3581 & & \\
\hline XMMU J140801.4-271340 & 54 & -13.68 & 40.39 & A3581 & & \\
\hline XMMU J140712.0-265007 & 51 & -13.94 & 40.24 & A3581 & ESO 510- G 065 & 0.025671 \\
\hline
\end{tabular}


Table B4. Properties of point sources studied in this work.

\begin{tabular}{|c|c|c|c|c|c|c|}
\hline Object Name & Net Counts & $\begin{array}{l}\text { Log Flux } \\
\operatorname{erg~cm}^{-2} \mathrm{~s}^{-1}\end{array}$ & $\begin{array}{l}\text { Log Luminosity } \\
\text { erg s }^{-1}\end{array}$ & Cluster & Optical Counterpart & Redshift \\
\hline XMMU J140840.1-270219 & 41 & -13.74 & 40.33 & A3581 & & \\
\hline XMMU J140800.7-270542 & 55 & -13.95 & 40.12 & A3581 & & \\
\hline XMMU J140844.4-270024 & 38 & -13.62 & 40.46 & A3581 & & \\
\hline XMMU J140652.8-265631 & 186 & -13.19 & 40.88 & A3581 & & \\
\hline XMMU J140806.5-270433 & 95 & -13.67 & 40.41 & A3581 & & \\
\hline XMMU J140809.4-265902 & 81 & -13.64 & 40.44 & A3581 & & \\
\hline XMMU J140752.1-264804 & 95 & -13.08 & 40.99 & A3581 & & \\
\hline XMMU J140757.1-265318 & 36 & -13.40 & 40.67 & A3581 & & \\
\hline XMMU J140741.5-265121 & 54 & -13.41 & 40.66 & A3581 & & \\
\hline XMMU J140650.2-270206 & 49 & -13.94 & 40.14 & A3581 & & \\
\hline XMMU J140645.6-265433 & 39 & -13.63 & 40.44 & A3581 & & \\
\hline XMMU J140750.9-270622 & 49 & -13.92 & 40.15 & A3581 & & \\
\hline XMMU J140632.6-270045 & 43 & -13.88 & 40.20 & A3581 & & \\
\hline XMMU J152305.3+083631 & 1237 & -12.71 & 41.72 & A2063 & CGCG 077-097 & 0.034174 \\
\hline XMMU J152252.6+083735 & 102 & -12.95 & 41.49 & $\mathrm{~A} 2063$ & & \\
\hline XMMU J152318.7+084319 & 67 & -13.73 & 40.72 & A2063 & & \\
\hline XMMU J152342.5+084535 & 64 & -13.22 & 41.23 & A2063 & & \\
\hline XMMU J152249.9+083643 & 201 & -13.28 & 41.16 & $\mathrm{~A} 2063$ & & \\
\hline XMMU J152323.5+083212 & 107 & -13.47 & 40.97 & A2063 & & \\
\hline XMMU J152331.2+082744 & 42 & -13.86 & 40.59 & A2063 & & \\
\hline XMMU J152326.9+083428 & 265 & -13.34 & 41.11 & A2063 & & \\
\hline XMMU J152250.9+084447 & 34 & -13.21 & 41.21 & A2063 & & \\
\hline XMMU J152256.6+083858 & 38 & -13.59 & 40.86 & $\mathrm{~A} 2063$ & 2MASX J15225650+0839004 & 0.03361 \\
\hline XMMU J152237.4+083530 & 42 & -13.59 & 40.85 & A2063 & & \\
\hline XMMU J152248.0+082759 & 87 & -13.61 & 40.84 & A2063 & & \\
\hline XMMU J152226.9+083556 & 35 & -13.93 & 40.52 & A2063 & & \\
\hline XMMU J152322.3+082159 & 46 & -13.22 & 41.23 & A2063 & & \\
\hline XMMU J152248.0+084306 & 44 & -13.94 & 40.51 & A2063 & & \\
\hline XMMU J152359.5+084117 & 32 & -13.55 & 40.90 & A2063 & & \\
\hline XMMU J152404.6+084115 & 31 & -13.59 & 40.86 & A2063 & & \\
\hline XMMU J152312.5+083259 & 1820 & -12.10 & 42.40 & $\mathrm{~A} 2063$ & 2MASX J15231224+0832590 & 0.036619 \\
\hline XMMU J152327.1+083553 & 53 & -13.22 & 41.27 & A2063 & & \\
\hline XMMU J152240.6+082621 & 47 & -13.28 & 41.20 & A2063 & & \\
\hline XMMU J152234.3+082854 & 31 & -13.94 & 40.55 & A2063 & & \\
\hline XMMU J152225.9+084134 & 30 & -13.92 & 40.57 & A2063 & & \\
\hline XMMU J152219.7+083803 & 94 & -13.59 & 40.90 & $\mathrm{~A} 2063$ & & \\
\hline XMMU J152224.7+084244 & 33 & -13.66 & 40.82 & A2063 & & \\
\hline XMMU J113449.4+490438 & 357 & -13.73 & 40.68 & A1314 & IC 712 & 0.03335 \\
\hline XMMU J113450.2+490326 & 344 & -13.76 & 40.65 & A1314 & & \\
\hline XMMU J113439.1+490623 & 53 & -13.95 & 40.46 & A1314 & & \\
\hline XMMU J113409.1+491516 & 48 & -12.22 & 42.28 & A1314 & 2MASX J11340896+4915162 & 0.03723 \\
\hline XMMU J113543.9+490215 & 273 & -12.13 & 42.15 & A1314 & MCG+08-21-065 & 0.02967 \\
\hline XMMU J113421.6+490050 & 334 & -13.47 & 40.95 & A1314 & & \\
\hline XMMU J113446.6+485721 & 200 & -13.64 & 40.72 & A1314 & IC 711 & 0.03160 \\
\hline XMMU J113447.3+490133 & 301 & -13.73 & 40.69 & A1314 & & \\
\hline XMMU J113359.3+490343 & 298 & -13.27 & 41.09 & A1314 & IC 708 & 0.03165 \\
\hline XMMU J113408.4+490318 & 207 & -13.16 & 41.24 & A1314 & & \\
\hline XMMU J113425.0+490647 & 111 & -13.50 & 40.90 & A1314 & & \\
\hline XMMU J113359.5+491246 & 69 & -13.76 & 40.65 & A1314 & LEDA 97398 & 0.031600 \\
\hline XMMU J113509.1+490658 & 91 & -13.88 & 40.54 & A1314 & & \\
\hline XMMU J113441.8+490918 & 118 & -13.86 & 40.56 & A1314 & & \\
\hline XMMU J113451.4+491203 & 137 & -13.56 & 42.36 & A1314 & & \\
\hline XMMU J113548.5+491150 & 49 & -13.48 & 40.93 & A1314 & & \\
\hline XMMU J113355.9+490955 & 61 & -13.32 & 41.08 & A1314 & & \\
\hline XMMU J113424.7+491230 & 38 & -13.85 & 40.55 & A1314 & & \\
\hline XMMU J113425.9+490909 & 81 & -13.83 & 40.59 & A1314 & & \\
\hline XMMU J113431.4+485354 & 34 & -13.91 & 40.50 & A1314 & & \\
\hline XMMU J113454.2+485506 & 30 & -13.87 & 40.53 & A1314 & & \\
\hline XMMU J113404.6+485519 & 76 & -13.38 & 41.02 & $\mathrm{~A} 1314$ & & \\
\hline XMMU J113331.2+485920 & 66 & -13.85 & 40.57 & A1314 & & \\
\hline XMMU J113412.2+485614 & 38 & -13.99 & 40.41 & A1314 & & \\
\hline XMMU J113420.9+490937 & 47 & -13.89 & 40.52 & A1314 & & \\
\hline XMMU J113424.7+491623 & 31 & -13.76 & 40.65 & A1314 & & \\
\hline XMMU J113526.2+485555 & 30 & -13.77 & 40.62 & A1314 & & \\
\hline XMMU J113533.8+485620 & 34 & -13.94 & 40.47 & A1314 & & \\
\hline XMMU J113317.3+490607 & 79 & -13.19 & 41.22 & A1314 & & \\
\hline
\end{tabular}


Table B5. Properties of point sources studied in this work.

\begin{tabular}{|c|c|c|c|c|c|c|}
\hline Object Name & Net Counts & $\begin{array}{c}\text { Log Flux } \\
\text { erg } \mathrm{cm}^{-2} \mathrm{~s}^{-1}\end{array}$ & $\begin{array}{l}\text { Log Luminosity } \\
\quad \operatorname{erg~s} \text { s }^{-1}\end{array}$ & Cluster & Optical Counterpart & Redshift \\
\hline XMMU J113328.3+491218 & 48 & -13.66 & 40.75 & A1314 & & \\
\hline XMMU J113517.8+491259 & 40 & -13.82 & 40.59 & A1314 & & \\
\hline XMMU J113334.6+485659 & 35 & -13.86 & 40.55 & A1314 & & \\
\hline XMMU J113347.3+490614 & 37 & -13.96 & 40.45 & $\mathrm{~A} 1314$ & & \\
\hline XMMU J113330.0+490901 & 31 & -13.77 & 40.63 & $\mathrm{~A} 1314$ & & \\
\hline XMMU J113423.0+490326 & 42 & -13.43 & 40.97 & A1314 & & \\
\hline XMMU J113408.6+485932 & 39 & -13.01 & 41.39 & A1314 & & \\
\hline XMMU J113406.5+490622 & 30 & -13.55 & 40.85 & A1314 & & \\
\hline XMMU J113539.8+485459 & 143 & -13.05 & 41.36 & A1314 & & \\
\hline XMMU J113554.7+485809 & 31 & -13.16 & 41.26 & A1314 & & \\
\hline XMMU J140141.8-113625 & 589 & -13.20 & 41.31 & A 1836 & MCG -02-36-002 & 0.037776 \\
\hline XMMU J140139.4-113749 & 197 & -13.37 & 41.11 & A1836 & & \\
\hline XMMU J140147.8-113438 & 31 & -13.65 & 40.81 & A1836 & & \\
\hline XMMU J140131.4-113309 & 37 & -13.81 & 40.66 & A1836 & & \\
\hline XMMU J140215.6-113748 & 417 & -12.63 & 41.84 & A1836 & & \\
\hline XMMU J140207.9-113553 & 411 & -13.42 & 41.06 & A1836 & & \\
\hline XMMU J140210.1-114131 & 164 & -13.90 & 40.59 & A1836 & & \\
\hline XMMU J140143.0-113059 & 85 & -13.23 & 41.23 & A1836 & & \\
\hline XMMU J140207.2-114351 & 62 & -13.75 & 40.73 & A1836 & & \\
\hline XMMU J140157.8-113927 & 71 & -13.21 & 41.06 & A1836 & & \\
\hline XMMU J140145.4-113133 & 66 & -13.64 & 40.84 & A1836 & & \\
\hline XMMU J140123.0-114535 & 29 & -13.94 & 40.54 & A1836 & & \\
\hline XMMU J140131.9-113925 & 31 & -13.46 & 41.12 & A1836 & 2MASX J14013206-1139261 & 0.041662 \\
\hline XMMU J140124.5-112832 & 30 & -13.81 & 40.67 & A1836 & & \\
\hline XMMU J140200.2-112752 & 94 & -13.64 & 40.84 & A1836 & & \\
\hline XMMU J140142.5-112754 & 84 & -13.63 & 40.86 & A1836 & & \\
\hline XMMU J140155.9-112952 & 101 & -13.81 & 40.67 & A1836 & & \\
\hline XMMU J140135.5-112708 & 32 & -13.39 & 41.05 & A1836 & & \\
\hline XMMU J140212.5-114443 & 33 & -13.58 & 40.88 & A1836 & & \\
\hline XMMU J140125.4-114717 & 39 & -13.51 & 40.96 & A1836 & & \\
\hline XMMU J140057.7-113746 & 32 & -13.46 & 41.00 & A1836 & & \\
\hline XMMU J140102.0-113316 & 69 & -13.46 & 41.12 & A1836 & & \\
\hline XMMU J140107.7-112915 & 34 & -13.44 & 41.14 & A1836 & & \\
\hline XMMU J140042.4-113447 & 39 & -13.03 & 41.56 & A1836 & & \\
\hline XMMU J140043.8-113731 & 38 & -13.42 & 41.18 & A1836 & & \\
\hline XMMU J140149.0-113837 & 501 & -12.91 & 41.70 & A1836 & & \\
\hline XMMU J140201.7-113408 & 51 & -13.56 & 41.03 & A1836 & & \\
\hline XMMU J140155.7-113808 & 54 & -13.91 & 40.59 & A1836 & 2MASX J14015570-1138043 & 0.036979 \\
\hline XMMU J140057.6-114051 & 49 & -13.68 & 40.82 & A1836 & & \\
\hline XMMU J140230.7-113646 & 42 & -13.80 & 40.70 & A1836 & & \\
\hline XMMU J140217.3-112612 & 29 & -13.91 & 40.59 & A1836 & & \\
\hline XMMU J140130.5-112247 & 37 & -13.72 & 40.78 & A1836 & & \\
\hline XMMU J011146.5-613139 & 445 & -13.92 & 40.29 & AS137 & NGC 0432 & 0.026929 \\
\hline XMMU J011142.8-613106 & 448 & -13.64 & 40.56 & AS137 & & \\
\hline XMMU J011205.4-613255 & 328 & -13.47 & 40.73 & AS137 & & \\
\hline XMMU J011208.8-613214 & 238 & -13.68 & 40.52 & AS137 & & \\
\hline XMMU J011150.0-613348 & 103 & -13.89 & 40.30 & AS137 & & \\
\hline XMMU J011134.8-613414 & 88 & -13.92 & 40.27 & AS137 & & \\
\hline XMMU J011142.1-612649 & 641 & -13.19 & 41.01 & AS137 & & \\
\hline XMMU J011211.4-612126 & 188 & -13.71 & 40.49 & AS137 & & \\
\hline XMMU J011014.3-613852 & 177 & -12.91 & 41.29 & AS137 & & \\
\hline XMMU J011120.3-612958 & 159 & -13.70 & 40.50 & AS137 & & \\
\hline XMMU J011222.9-612811 & 104 & -13.81 & 40.39 & AS137 & & \\
\hline XMMU J011116.1-612822 & 122 & -13.98 & 40.21 & AS137 & & \\
\hline XMMU J011123.1-612054 & 83 & -13.34 & 40.85 & AS137 & & \\
\hline XMMU J011127.4-612612 & 104 & -13.49 & 40.70 & AS137 & & \\
\hline XMMU J011210.5-612807 & 110 & -13.99 & 40.21 & AS137 & & \\
\hline XMMU J011207.4-614033 & 99 & -13.95 & 40.25 & AS137 & & \\
\hline XMMU J011109.0-613049 & 81 & -13.49 & 40.70 & AS137 & & \\
\hline XMMU J011115.6-613242 & 111 & -13.65 & 40.55 & AS137 & & \\
\hline XMMU J011211.3-612439 & 92 & -13.53 & 40.66 & AS137 & & \\
\hline XMMU J011128.8-613519 & 94 & -13.71 & 40.48 & AS137 & & \\
\hline XMMU J011154.6-614035 & 62 & -13.73 & 40.46 & AS137 & & \\
\hline XMMU J011136.7-612338 & 79 & -13.90 & 40.29 & AS137 & & \\
\hline XMMU J011144.0-614135 & 38 & -13.79 & 40.40 & AS137 & & \\
\hline XMMU J011325.0-612746 & 67 & -13.99 & 40.21 & AS137 & & \\
\hline XMMU J011201.8-612157 & 55 & -13.89 & 40.30 & AS137 & & \\
\hline
\end{tabular}


Table B6. Properties of point sources studied in this work.

\begin{tabular}{|c|c|c|c|c|c|c|}
\hline Object Name & Net Counts & $\begin{array}{l}\log \text { Flux } \\
\mathrm{erg} \mathrm{cm}^{-2} \mathrm{~s}^{-1}\end{array}$ & $\begin{array}{l}\text { Log Luminosity } \\
\operatorname{erg~s}^{-1}\end{array}$ & Cluster & Optical Counterpart & Redshift \\
\hline XMMU J011105.5-612548 & 141 & -13.02 & 41.16 & AS137 & & \\
\hline XMMU J010949.4-613153 & 174 & -13.06 & 41.13 & AS137 & & \\
\hline XMMU J011220.2-613752 & 83 & -13.57 & 40.62 & AS137 & & \\
\hline XMMU J011059.5-613832 & 52 & -13.94 & 40.25 & AS137 & & \\
\hline XMMU J011114.5-612922 & 48 & -13.78 & 40.41 & AS137 & & \\
\hline XMMU J011251.4-613959 & 65 & -13.77 & 40.43 & AS137 & 2MASX J01125179-6139513 & 0.026442 \\
\hline XMMU J011058.3-612752 & 107 & -13.48 & 40.71 & AS137 & & \\
\hline XMMU J011318.7-613133 & 64 & -13.33 & 40.86 & AS137 & & \\
\hline XMMU J011022.1-613043 & 46 & -13.70 & 40.49 & AS137 & & \\
\hline XMMU J011234.0-613615 & 29 & -13.73 & 40.46 & AS137 & & \\
\hline XMMU J011054.8-612940 & 42 & -13.83 & 40.36 & AS137 & & \\
\hline XMMU J011315.5-613609 & 47 & -13.67 & 40.52 & AS137 & & \\
\hline XMMU J011029.9-612637 & 37 & -13.92 & 40.27 & AS137 & & \\
\hline XMMU J011244.1-612341 & 30 & -13.71 & 40.48 & AS137 & & \\
\hline XMMU J011255.6-613655 & 31 & -13.62 & 40.57 & AS137 & & \\
\hline XMMU J011249.7-612938 & 39 & -13.89 & 40.30 & AS137 & & \\
\hline XMMU J011340.9-613101 & 38 & -13.49 & 40.70 & AS137 & & \\
\hline XMMU J011247.9-613645 & 35 & -13.95 & 40.25 & AS137 & & \\
\hline XMMU J011157.1-613920 & 31 & -13.89 & 40.30 & AS137 & & \\
\hline XMMU J011048.5-614303 & 30 & -13.72 & 40.48 & AS137 & & \\
\hline XMMU J011202.3-613006 & 93 & -13.47 & 40.72 & AS137 & & \\
\hline XMMU J011142.8-612813 & 85 & -13.69 & 40.51 & AS137 & & \\
\hline XMMU J011213.1-612015 & 81 & -13.36 & 40.84 & AS137 & & \\
\hline XMMU J011314.2-612331 & 65 & -13.41 & 40.78 & AS137 & & \\
\hline XMMU J011231.9-611906 & 37 & -13.55 & 40.64 & AS137 & & \\
\hline XMMU J011220.4-613648 & 50 & -13.65 & 40.55 & AS137 & & \\
\hline XMMU J011314.3-612249 & 37 & -13.46 & 40.73 & AS137 & & \\
\hline XMMU J010959.0-612601 & 28 & -13.46 & 40.73 & AS137 & & \\
\hline XMMU J011007.3-612417 & 30 & -13.71 & 40.49 & AS137 & & \\
\hline XMMU J141220.9-342022 & 1460 & -13.83 & 40.72 & AS758 & MCG-06-31-029 & 0.03850 \\
\hline XMMU J141117.5-341116 & 302 & -13.09 & 41.42 & AS758 & & \\
\hline XMMU J141216.6-342422 & 988 & -13.06 & 41.46 & AS758 & & \\
\hline XMMU J141226.2-341715 & 746 & -13.49 & 41.03 & AS758 & & \\
\hline XMMU J141201.2-341548 & 430 & -13.50 & 41.03 & AS758 & & \\
\hline XMMU J141308.6-342105 & 197 & -13.01 & 41.49 & AS758 & & \\
\hline XMMU J141200.2-341657 & 272 & -13.85 & 40.68 & AS758 & & \\
\hline XMMU J141157.6-341840 & 303 & -13.92 & 40.61 & AS758 & & \\
\hline XMMU J141231.7-342141 & 363 & -13.79 & 40.74 & AS758 & & \\
\hline XMMU J141124.7-342353 & 248 & -13.59 & 40.94 & AS758 & & \\
\hline XMMU J141253.8-342638 & 243 & -13.85 & 40.68 & AS758 & & \\
\hline XMMU J141312.2-342051 & 123 & -13.69 & 40.83 & AS758 & & \\
\hline XMMU J141200.0-342245 & 115 & -13.91 & 40.61 & AS758 & & \\
\hline XMMU J141216.8-342556 & 112 & -13.98 & 40.54 & AS758 & & \\
\hline XMMU J141246.8-342532 & 139 & -13.80 & 40.72 & AS758 & & \\
\hline XMMU J141145.6-342441 & 177 & -13.89 & 40.64 & AS758 & & \\
\hline XMMU J141200.7-341036 & 123 & -13.67 & 40.85 & AS758 & & \\
\hline XMMU J141311.3-342216 & 281 & -13.69 & 40.84 & AS758 & & \\
\hline XMMU J141229.3-341741 & 149 & -13.96 & 40.67 & AS758 & 2MASX J14122917-3417417 & 0.04300 \\
\hline XMMU J141134.3-342353 & 71 & -13.89 & 40.63 & AS758 & & \\
\hline XMMU J141210.3-343142 & 64 & -13.31 & 41.20 & AS758 & & \\
\hline XMMU J141259.5-341734 & 93 & -13.79 & 40.72 & AS758 & & \\
\hline XMMU J141241.3-342659 & 135 & -13.80 & 40.72 & AS758 & & \\
\hline XMMU J141149.9-343008 & 102 & -13.90 & 40.63 & AS758 & & \\
\hline XMMU J141127.8-342255 & 66 & -13.81 & 40.71 & AS758 & & \\
\hline XMMU J141246.3-342816 & 63 & -13.71 & 40.80 & AS758 & & \\
\hline XMMU J141314.2-341952 & 88 & -13.62 & 40.89 & AS758 & & \\
\hline XMMU J141215.8-342717 & 30 & -13.74 & 40.77 & AS758 & & \\
\hline XMMU J141249.7-342552 & 62 & -13.82 & 40.69 & AS758 & & \\
\hline XMMU J141120.2-341449 & 63 & -13.55 & 40.96 & AS758 & & \\
\hline XMMU J141207.7-340830 & 67 & -13.88 & 40.64 & AS758 & & \\
\hline XMMU J141232.8-341653 & 70 & -13.97 & 40.54 & AS758 & & \\
\hline XMMU J141118.2-342059 & 35 & -13.68 & 40.82 & AS758 & & \\
\hline XMMU J141230.7-340947 & 757 & -13.56 & 40.98 & AS758 & & \\
\hline XMMU J141255.7-341247 & 362 & -13.78 & 40.75 & AS758 & & \\
\hline XMMU J141216.6-341034 & 179 & -13.50 & 41.01 & AS758 & & \\
\hline XMMU J141301.7-341331 & 63 & -13.85 & 40.67 & AS758 & & \\
\hline XMMU J141241.6-341103 & 119 & -13.90 & 40.62 & AS758 & & \\
\hline
\end{tabular}


Table B7. Properties of point sources studied in this work.

\begin{tabular}{|c|c|c|c|c|c|c|}
\hline Object Name & Net Counts & $\begin{array}{c}\text { Log Flux } \\
\mathrm{erg} \mathrm{cm}^{-2} \mathrm{~s}^{-1}\end{array}$ & $\begin{array}{l}\text { Log Luminosity } \\
\operatorname{erg~s}^{-1}\end{array}$ & Cluster & Optical Counterpart & Redshift \\
\hline XMMU J141146.6-341906 & 87 & -13.86 & 40.65 & AS758 & & \\
\hline XMMU J141219.9-342557 & 89 & -13.86 & 40.65 & AS758 & & \\
\hline XMMU J141144.2-341353 & 74 & -13.88 & 40.63 & AS758 & & \\
\hline XMMU J141209.8-341327 & 55 & -13.71 & 40.80 & AS758 & & \\
\hline XMMU J141139.1-340615 & 79 & -13.50 & 41.02 & AS758 & & \\
\hline XMMU J141117.0-342710 & 74 & -13.68 & 40.84 & AS758 & & \\
\hline XMMU J141204.1-340538 & 42 & -13.37 & 41.13 & AS758 & & \\
\hline XMMU J141221.6-342716 & 52 & -13.96 & 40.56 & AS758 & & \\
\hline XMMU J141139.6-340810 & 72 & -13.77 & 40.75 & AS758 & & \\
\hline XMMU J141141.0-340707 & 39 & -13.77 & 40.75 & AS758 & & \\
\hline XMMU J141137.0-340736 & 38 & -13.36 & 41.15 & AS758 & & \\
\hline XMMU J141236.7-342930 & 54 & -13.95 & 40.57 & AS758 & & \\
\hline XMMU J141302.4-342438 & 37 & -13.86 & 40.64 & AS758 & & \\
\hline XMMU J141311.8-341709 & 40 & -13.75 & 40.76 & AS758 & & \\
\hline XMMU J141234.3-341849 & 151 & -13.58 & 40.93 & AS758 & & \\
\hline XMMU J141240.1-341617 & 56 & -13.64 & 40.88 & AS758 & & \\
\hline XMMU J141245.4-342343 & 343 & -13.35 & 41.18 & AS758 & & \\
\hline XMMU J141304.3-342557 & 43 & -13.44 & 41.07 & AS758 & & \\
\hline XMMU J141215.6-341628 & 42 & -13.98 & 40.54 & AS758 & & \\
\hline XMMU J141149.9-341643 & 46 & -13.95 & 40.57 & AS758 & & \\
\hline XMMU J141300.2-342947 & 29 & -13.91 & 40.61 & AS758 & & \\
\hline XMMU J141223.0-341330 & 218 & -13.47 & 41.06 & AS758 & & \\
\hline XMMU J231638.6-022527 & 522 & -12.54 & 41.67 & RXCJ2315.7-0222 & & \\
\hline XMMU J231544.4-022254 & 1768 & -13.61 & 40.56 & RXCJ2315.7-0222 & NGC 7556 & 0.025041 \\
\hline XMMU J231624.5-021457 & 310 & -13.35 & 40.80 & RXCJ2315.7-0222 & & \\
\hline XMMU J231553.8-022615 & 235 & -13.78 & 40.38 & RXCJ2315.7-0222 & & \\
\hline XMMU J231623.8-022550 & 132 & -13.54 & 40.61 & RXCJ2315.7-0222 & & \\
\hline XMMU J231523.5-021660 & 130 & -13.67 & 40.48 & RXCJ2315.7-0222 & & \\
\hline XMMU J231637.4-021950 & 110 & -13.83 & 40.32 & RXCJ2315.7-0222 & NGC 7566 & 0.026548 \\
\hline XMMU J231535.8-021727 & 71 & -13.99 & 40.16 & RXCJ2315.7-0222 & & \\
\hline XMMU J231555.9-021644 & 85 & -13.33 & 40.81 & RXCJ2315.7-0222 & & \\
\hline XMMU J231610.3-021128 & 74 & -13.80 & 40.35 & RXCJ2315.7-0222 & & \\
\hline XMMU J231605.0-022533 & 59 & -13.95 & 40.20 & RXCJ2315.7-0222 & & \\
\hline XMMU J231527.6-021525 & 60 & -13.56 & 40.58 & RXCJ2315.7-0222 & & \\
\hline XMMU J231618.2-021330 & 37 & -13.58 & 40.57 & RXCJ2315.7-0222 & & \\
\hline XMMU J231610.3-021502 & 29 & -13.47 & 40.67 & RXCJ2315.7-0222 & & \\
\hline XMMU J231610.3-021238 & 36 & -13.76 & 40.39 & RXCJ2315.7-0222 & & \\
\hline XMMU J231600.0-022943 & 159 & -13.23 & 40.91 & RXCJ2315.7-0222 & & \\
\hline XMMU J231619.7-021351 & 50 & -13.62 & 40.53 & RXCJ2315.7-0222 & & \\
\hline XMMU J231552.3-023008 & 73 & -13.76 & 40.39 & RXCJ2315.7-0222 & & \\
\hline XMMU J231547.8-022841 & 86 & -13.94 & 40.21 & RXCJ2315.7-0222 & & \\
\hline XMMU J231631.4-021247 & 471 & -12.88 & 41.33 & RXCJ2315.7-0222 & & \\
\hline XMMU J231642.2-022452 & 114 & -12.87 & 41.26 & RXCJ2315.7-0222 & & \\
\hline XMMU J231504.6-022254 & 59 & -13.62 & 40.53 & RXCJ2315.7-0222 & & \\
\hline XMMU J231559.0-021042 & 31 & -13.69 & 40.46 & RXCJ2315.7-0222 & & \\
\hline XMMU J231614.4-021426 & 45 & -13.97 & 40.18 & RXCJ2315.7-0222 & & \\
\hline XMMU J231544.6-023059 & 44 & -13.82 & 40.32 & RXCJ2315.7-0222 & & \\
\hline XMMU J231538.9-021608 & 37 & -13.60 & 40.54 & RXCJ2315.7-0222 & & \\
\hline XMMU J231633.8-023017 & 35 & -13.67 & 40.48 & RXCJ2315.7-0222 & & \\
\hline XMMU J231500.7-022515 & 91 & -13.36 & 40.85 & RXCJ2315.7-0222 & & \\
\hline XMMU J231602.2-022532 & 47 & -13.83 & 40.38 & RXCJ2315.7-0222 & & \\
\hline XMMU J231513.7-022235 & 31 & -13.74 & 40.46 & RXCJ2315.7-0222 & & \\
\hline XMMU J231527.1-022812 & 38 & -13.65 & 40.55 & RXCJ2315.7-0222 & & \\
\hline XMMU J231610.3-023034 & 29 & -13.58 & 40.62 & RXCJ2315.7-0222 & & \\
\hline XMMU J231547.8-023050 & 45 & -13.95 & 40.26 & RXCJ2315.7-0222 & & \\
\hline XMMU J231556.6-022415 & 30 & -13.54 & 40.65 & RXCJ2315.7-0222 & & \\
\hline
\end{tabular}

This paper has been typeset from a $\mathrm{T}_{\mathrm{E}} \mathrm{X} / \mathrm{LAT}_{\mathrm{E}} \mathrm{X}$ file prepared by the author. 